\title{
The Low-Temperature Behavior of Disordered Magnets
}

\author{
J. T. Chayes ${ }^{1, \star}$, L. Chayes ${ }^{1, \star}$, and J. Fröhlich ${ }^{2, \star \star}$ \\ 1 Departments of Mathematics and Physics, Harvard University, Cambridge, MA 02138, USA \\ 2 The Institute for Advanced Study, Princeton, NJ 08540, USA
}

\begin{abstract}
We map out the low-temperature phase diagrams of dilute Ising ferromagnets and predominantly ferromagnetic ferrites, obtaining nonperturbative and essentially optimal conditions on the density of ferromagnetic couplings required to maintain long-range order. We also study mappings of dilute antiferromagnets in a uniform field onto random field ferromagnets.

For the randomly dilute systems, we prove that ferromagnetically ordered states exist at low temperature if the density of ferromagnetic couplings exceeds the (appropriately defined) percolation threshold, thereby extending the result of Georgii to three or more dimensions. We also show that, for these systems, as the temperature tends to zero, the magnetization approaches the percolation probability of the corresponding Bernoulli system. In two dimensions, we prove that low-temperature ordering persists in the presence of antiferromagnetic impurities if the ferromagnetic couplings percolate and if the density of antiferromagnetic couplings is bounded above by the order of the inverse square of the corresponding percolation correlation length. For these systems, we rigorously compute the first order decrease in the zerotemperature nominal spontaneous magnetization, in terms of derivatives of the percolation probability, thereby establishing the existence of ferrimagnetically ordered states. Finally, we introduce a model of a random ferrite which exhibits spontaneous magnetization anticorrelated with the boundary conditions.
\end{abstract}

\section{Organization and Main Results}

In this paper we study the phase diagrams and order parameters of disordered Ising magnets. Our main rigorous results concern dilute, predominantly fer-

* National Science Foundation Postdoctoral Research Fellows. Work supported in part by the National Science Foundation under Grant No. PHY-8203669

$\star \star$ Work supported in part by the National Science Foundation under Grant No. MCS-8108814 (A03)

Permanent address: Theoretical Physics, ETH-Hönggerberg, CH-8093 Zürich, Switzerland 
romagnetic Ising magnets at low temperatures, but we also discuss effects such as anticorrelation and frustration and reconsider random antiferromagnets in a uniform magnetic field.

The magnetic systems studied below have the following features: At each site, $j$, of a regular lattice, henceforth chosen to be $\mathbb{Z}^{d}$, there is an Ising spin, $\sigma_{j}$, with values \pm 1 . If $\Lambda$ is some finite subset of $\mathbb{Z}^{d}$, we denote by $\sigma_{\Lambda}$ the configuration of spins $\sigma_{j}$, with $j \in \Lambda$, and by $\sigma_{\Lambda^{c}}$ the configuration of $\sigma_{i}, i \in \Lambda^{c}$. by

The Hamilton function, or energy, of a configuration $\sigma_{\Lambda}$, with $\sigma_{\Lambda^{c}}$ fixed, is given

$$
\mathscr{H}\left(\sigma_{\Lambda}, \sigma_{\Lambda^{c}}\right)=-\sum_{i, j: j \in \Lambda} J_{i j} \sigma_{i} \sigma_{j}-\sum_{j} h_{j} \sigma_{j}
$$

where the exchange couplings $J_{i j}$ are random variables with distribution

$$
d R(J)=\prod_{i j} d \varrho_{i j}\left(J_{i j}\right)
$$

and $d \varrho_{i j}$ is a probability measure depending only on $i-j$. Typically,

$$
d \varrho_{i j}(J)= \begin{cases}d \varrho(J), & |i-j|=1, \\ \delta(J) d J, & \text { otherwise },\end{cases}
$$

where $d \varrho$ is a probability measure favoring ferromagnetic couplings. The distribution (1.2) describes systems with bond disorder. Another popular choice is site disorder, as given by

$$
J_{i j}= \begin{cases}J \tau_{i} \tau_{j}, & |i-j|=1, \\ 0, & \text { otherwise }\end{cases}
$$

where $\left\{\tau_{j}\right\}_{j \in \mathbb{Z}^{d}}$ are i.i.d. random variables, and, by convention, $J>0$. Although, for definiteness, we shall restrict attention to random bond systems, most of our results go through mutatis mutandis for systems with site randomness.

The variables $\left\{h_{j}\right\}_{j \in \mathbb{Z}^{d}}$ are local magnetic fields which will generally be chosen as follows:

(a) $h_{j}=0$, for all $j$, or

(b) $h_{j}=h>0$, for all $j$, or

(c) the $h_{j}$ 's are i.i.d. random variables with mean 0 and variance $H>0$.

The joint distribution of the magnetic field variables is denoted by $d L(h)$ and is a product measure in all three cases.

We note that the Hamilton function $\mathscr{H}$ is really a random function, as it depends on $\left\{J_{i j}\right\}$ and $\left\{h_{j}\right\}$. The equilibrium expectation of a function, $F$, of the spins $\sigma_{A}$ in a system with Hamilton function given by (1.1), where $J=\left\{J_{i j}\right\}$ and $h=\left\{h_{j}\right\}$ are arbitrary, but fixed samples, and with boundary condition $\alpha=\alpha\left(\sigma_{\Lambda^{c}}\right)$ imposed on the spins outside $\Lambda$, is given by

$$
\langle F\rangle_{\beta, \Lambda^{\alpha}}(J, h) \equiv Z_{\beta, \Lambda^{\alpha}}(J, h)^{-1} \sum_{\sigma_{\Lambda}, \sigma_{\Lambda^{c}}} e^{-\beta \mathscr{H}\left(\sigma_{\Lambda}, \sigma_{\Lambda^{c}}\right)} F(\sigma) \alpha\left(\sigma_{\Lambda^{c}}\right),
$$

where $\beta$ is the inverse temperature, and the partition function, $Z_{\beta, \Lambda^{\alpha}}(J, h)$, is chosen such that

$$
\langle 1\rangle_{\beta, \Lambda^{\alpha}}(J, h)=1
$$


Clearly, $\langle F\rangle_{\beta, \Lambda^{\alpha}}(J, h)$ and $Z_{\beta, \Lambda^{\alpha}}(J, h)$ are random variables through their dependence on $J, h$. We define the free energy per site by

$$
f(\beta, h ; R, L) \equiv \lim _{\Lambda \mp \mathbb{Z}^{d}}-\frac{1}{\beta|\Lambda|} \log Z_{\beta, \Lambda^{\alpha}}(J, h),
$$

where $|\Lambda|$ denotes the number of sites in $\Lambda$ (and $\Lambda \uparrow \mathbb{Z}^{d}$ e.g. in the sense of van Hove). It is well known [1] that, for couplings $J_{i j}$ of not too long range, the limit in (1.5) exists, is independent of the b.c. $\alpha$ and is almost surely (a.s.) constant in $J$ and $h$ [i.e. independent of the particular sample. It does, of course, depend on the distributions $d R(J)$ and $d L(h)$ ]. There are other quantities which, under suitable hypotheses, are sample-independent, e.g. the correlation length or the spontaneous magnetization, but local correlation functions $d o$, of course, depend on $J$ and $h$. This motivates defining quenched expectations:

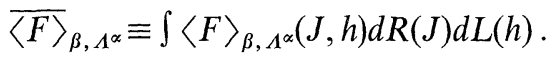

The spontaneous magnetization is defined by

$$
m(\beta)=\lim _{h \searrow 0} \frac{\partial f(\beta, h ; R, L)}{\partial h},
$$

and a variant of a result in [2] yields

$$
m(\beta)=\lim _{\Lambda \rtimes \mathbb{Z}^{d}} \max _{\alpha}|\Lambda|^{-1} \sum_{j \in \Lambda}\left\langle\sigma_{j}\right\rangle_{\beta, \Lambda^{\alpha}}(J) .
$$

Finally, the Edwards-Anderson order parameter [3] is given by [2]

$$
q_{\mathrm{EA}}(\beta)=\lim _{\Lambda \ni \mathbb{Z}^{d}} \max _{\alpha}|\Lambda|^{-1} \sum_{j \in \Lambda}\left[\left\langle\sigma_{j}\right\rangle_{\beta, \Lambda^{\alpha}}(J, h)\right]^{2} .
$$

We now proceed to summarize our main results.

In Sect. 2, we analyze a simple example of a predominantly ferromagnetic random Ising model in zero magnetic field. The exchange couplings $J_{i j}$ are distributed as in (1.2), (1.3), with the distribution de given, for example, by

$$
d \varrho(J)=(\sqrt{2 \pi} \Delta)^{-1} \exp \left[-(J-\bar{J})^{2} / 2 \Delta^{2}\right] d J .
$$

For $\bar{J}$ large enough and/or $\Delta$ small enough, we show that on a set of exchange couplings of $d R$-measure close to 1 , the Peierls-Griffiths estimate $[4,5]$ converges if + boundary conditions are imposed on the boundary, $\partial \Lambda$, of $\Lambda$ (i.e., $\sigma_{j}=+1$, $\forall j \in \Lambda^{c}$ ). This enables us to prove positivity of the magnetization for $\beta$ large enough. (Although this argument is quite simple, it does not - to our surprise - seem to have appeared in the literature.)

Unfortunately, the straightforward arguments of Sect. 2 do not provide optimal, or even necessarily good bounds on the values of the parameters $\bar{J}$ (the mean of $J_{i j}$ ), $\Delta$ (the spread of $J_{i j}$ ), and $\beta$ at which ferromagnetic ordering disappears. We, therefore, turn attention to a simple, special example for which one can find some optimal results, and which is, moreover, experimentally realizable (as e.g. $\mathrm{Rb}_{2} \mathrm{CO}_{p} \mathrm{Mg}_{1-p} \mathrm{~F}_{4}$, see [6-8]). This model is the dilute Ising ferromagnet with exchange couplings, $J_{i j}$, given by

$$
J_{i j}= \begin{cases}J=J_{F}>0, & \text { with probability } p, \\ 0, & \text { with probability }(1-p),\end{cases}
$$


if $|i-j|=1$, and $J_{i j}=0$, otherwise. For this model, we can derive essentially optimal results on the parameter $p$ in all dimensions.

The couplings above are distributed as in (1.2), (1.3), with $d R$ here being the Bernoulli bond percolation measure, $d \mu_{p}$, at density $p$. We say that a bond $\langle i, j\rangle$ is ferromagnetic, or occupied, if $J_{i j}>0$, which happens with probability $p$; otherwise, it is empty.

The theoretical status of dilute Ising ferromagnets with Bernoulli coupling distribution may be found in any of several fine reviews [9-12]. The system was first analyzed rigorously by Griffiths and Lebowitz [1], who showed that, when $p$ is large enough, there is ordering at low temperatures. One rightly expects that the correct condition is $p>p_{c}$; that is, if $p$ exceeds the percolation threshold, the model should exhibit spontaneous magnetization at low temperature. Such a result has been proved in $d=2$, by Georgii [13].

Since the appearance of ferromagnetic order at low temperature depends, in a crucial way, on the structure of ferromagnetic clusters, and since the ferromagnetic bonds are distributed according to the Bernoulli percolation measure, we shall need to review some results of percolation theory. This is done in Sect. 3. In particular, we review (and slightly extend) the results of [14] to show that in two or more dimensions and for densities, $p$, of ferromagnetic bonds above the (appropriately defined) percolation threshold, there a.s. exists an infinite cluster of ferromagnetic bonds containing a subset with the structure of a coarse-grained, at least two-dimensional lattice of renormalized bonds. Only a small fraction of the renormalized bonds are broken, and this fraction vanishes with the degree of bond rescaling. This result is intuitively clear in two dimensions. However, in higher dimensions, the renormalized bonds may miss each other, even if their projections onto two-dimensional lattice planes intersect. This difficulty can be overcome by studying percolation of bonds in slabs of the form

$$
\mathbb{Z}^{2} \times\{0, \ldots, k\}^{d-2},
$$

with $k$ chosen large enough, depending on $p$, and by using the fact that for $p$ above the (appropriately defined) threshold, the infinite cluster in such slabs is a.s. unique [14].

In Sects. 4 and 5, we combine the results just described with a Peierls argument and some fairly standard combinatorial estimates to prove that if the density, $p$, of ferromagnetic bonds exceeds the appropriate percolation threshold, then the spontaneous magnetization is positive at low enough temperatures, depending on $p$. We also prove the widely believed conjecture that as the temperature tends to zero, the spontaneous magnetization approaches the probability of connection to infinity in the corresponding Bernoulli percolation system. This is, of course, a prerequisite to establishing a crossover from thermal to percolative behavior in dilute magnetic systems.

The above results show that percolation of ferromagnetism is sufficient to achieve ferromagnetic ordering at low temperature. That it is necessary is trivial to verify. Indeed, if $p$ is below the percolation threshold, then almost surely all connected clusters of ferromagnetic bonds are finite, and hence the Gibbs state is a.s. unique at all temperatures; in particular, there is no spontaneous magnetiza- 
tion. It is well known that in a homogeneous magnetic field $h \neq 0$ there are no phase transitions and the Gibbs state is a.s. unique [15].

The above results, combined with previously known properties described below, give us a fairly detailed picture of the phase diagram of dilute ferromagnets. Let $T_{c}(p)$ denote the critical temperature of the model described above, with $T_{c}(1)$ the transition temperature of the pure Ising model. Modulo the usual conjectures of rigorous percolation theory, we now know that

$$
\begin{aligned}
& T_{c}(p)=0 \text {, for } p<p_{c}, \\
& T_{c}(p)>0 \text {, for } p>p_{c} \text {, }
\end{aligned}
$$

in two or more dimensions. Here $p_{c}$ is the percolation threshold. The usual conjectures referred to above are simply that in $d \geqq 3, p_{c}$ coincides with the limit of thresholds for slabs of the form (1.11). The critical temperature $T_{c}(p)$ in (1.12) may be related to the Ising critical temperature through the upper bound of Falk and Gehring [16]:

$$
T_{c}(p)<p T_{c}(1) .
$$

The bound (1.13) can also be proved by using the correlation inequalities due to Olivieri [17]. Improved upper bounds are given by Harris [18], and much improved upper (and lower) bounds are given in the paper of Bergstresser [19]. Putting these together with our results (1.12), the phase diagram of the system may be summarized as in Fig. 1, below.

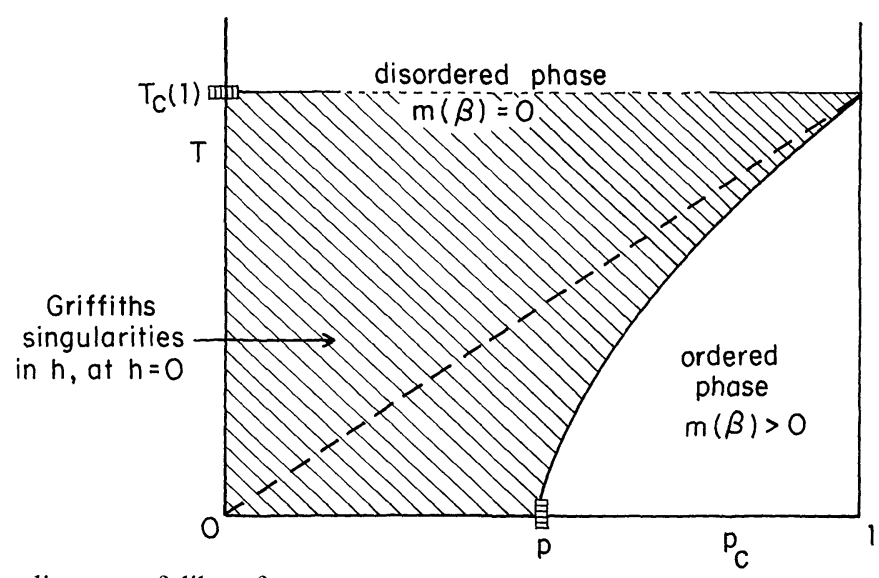

Fig. 1. Phase diagram of dilute ferromagnet

In order to complete the (rigorously known) phase diagram, let us briefly mention several other results. First, as was shown by Griffiths [20], the free energy $f(\beta, h ; p)$ of the dilute magnet, as a function of the magneticfield $h$, has an essential singularity (now termed a Griffiths singularity) at $h=0$ for all $p \in(0,1)$ and $T<T_{c}(1)$. These singularities cause the high temperature expansion to diverge at temperatures well above $T_{c}(p)$. (See [21] for a discussion of these singularities and for an improved high temperature expansion which converges for temperatures below $T_{c}(1)$ but still well above $T_{c}(p)$.) Second, we note that by using the Lebowitz 
inequality [22], it can be shown that the susceptibility tends continuously to $\infty$ as $T \downarrow T_{c}(p)$. Finally, for $d=2$, Georgii [23] has recently obtained very accurate bounds on the rate at which $T_{c}(p)$ tends to zero as $p \searrow p_{c}$. (See also [19].)

Given the detailed picture of the phase diagram and critical behavior of dilute ferromagnets described above, it is natural to ask whether this picture remains stable when a small density of antiferromagnetic bonds are introduced. This question is addressed in Sect. 6. It turns out that in two dimensions we can give a much more detailed answer than in higher dimensions, so two-dimensional models are discussed first. Consider an Ising model with nearest neighbor exchange couplings $J_{i j}$ distributed as follows

$$
J_{i j}=\left\{\begin{array}{cl}
J_{F}, & \text { with probability } p, \\
0, & \text { with probability } 1-p-\varepsilon, \\
-J_{A}, & \text { with probability } \varepsilon,
\end{array}\right.
$$

with $J_{F}>0, J_{A}>0$. We must distinguish three cases:

(1) $p+\varepsilon<p_{c}$,

(2) $p<p_{c}, \varepsilon<p_{c}, p+\varepsilon>p_{c}$,

(3) $p>p_{c}, \varepsilon<p_{c}$.

In three or more dimensions, there is also a fourth case, $p>p_{c}$ and $\varepsilon>p_{c}$, but this is excluded in two dimensions, since $p_{c}=1 / 2$ [24].

In case 1 , the Gibbs state is a.s. unique for all $\beta$ and $h$, since there are a.s. only finite connected clusters of nonzero $J_{i j}$ 's. Case 2 is very difficult! Depending on the values of the parameters, it describes systems ranging from random field Ising models (for $h \neq 0$ ) to spin glasses. Some heuristic considerations may be found in Sect. 6.C(ii). Since in this case local ordering and frustration compete, fairly complex behavior must be expected.

Our principal results concern case 3 . This model has, almost surely, an infinite ferromagnetic cluster. The first question we address is whether, and under what circumstances this infinite ferromagnetic cluster tends to align with the direction prescribed by the boundary conditions, and hence magnetize. While the analogous question was straightforward in the case of dilute ferromagnets, it is clear that the presence of antiferromagnetic bonds can tend to disorder the ferromagnetic infinite cluster. Among our results is a bound on the critical antiferromagnetic tolerance, i.e. the density of antiferromagnetic bonds which can be present without destroying the magnetization of the infinite cluster at low temperatures. Let $\xi(p)$ denote the correlation length of the pure Bernoulli percolation system at density $p$. We prove (see Theorem 6.3) that the infinite ferromagnetic cluster maintains order at low enough temperature if

$$
\frac{J_{A}}{J_{F}} \varepsilon \leqq O\left(\xi^{-2}(p)\right)
$$

Assuming the unproven conjecture that $\xi(p)$ has an associated critical exponent, $v$, this provides a bound in terms of $v$ on the antiferromagnetic tolerance as $p \searrow p_{c}$. A consequence of magnetization of the infinite cluster is

$$
q_{\mathrm{EA}}(\beta)>0
$$


for $p>p_{c}, \varepsilon$ satisfying (1.15) and $\beta$ large enough (depending on $p$ and $\varepsilon$ ). In three or more dimensions we can, for technical reasons, prove (1.16) only for fairly large (but nevertheless nonperturbative) values of $p$. Although our higher dimensional results are not optimal, they do represent an improvement over other bounds [25, $26]$ on the critical $p$ for systems with antiferromagnetism.

Next we examine the effect of finite ferromagnetic clusters. Many of these will be coupled to the infinite ferromagnetic cluster by antiferromagnetic bonds. Heuristically, one can see that, if $\varepsilon$ is small enough so that most finite clusters which are connected to the infinite cluster at all are actually connected through a single antiferromagnetic bond, then the majority of the spins so connected will tend to align in the direction opposite to the one prescribed by the boundary conditions. This makes clear that the square of the spontaneous magnetization may be considerably smaller than the Edwards-Anderson order parameter, and that pronounced hysteresis phenomena and "path-dependent behavior" may arise as an external field is varied.

Our next result addresses the effect of the antiferromagnetism on the magnetization. To simplify the discussion, we take $J_{F}>J_{A}$ so that frustration effects are less severe. Let $P_{\infty}(p)$ denote the density of sites belonging to the infinite cluster in the pure Bernoulli percolation system at density $p$. We show (see Lemma 6.4 and Theorem 6.5) that

$$
\lim _{\beta \rightarrow \infty} m(\beta) \geqq P_{\infty}(p)-\varepsilon \frac{d P_{\infty}}{d p}+o(\varepsilon) .
$$

Moreover, if $m^{+}(\beta)$ is the spontaneous magnetization in the state with + boundary conditions, then

$$
\lim _{\beta \rightarrow \infty} m^{+}(\beta)=P_{\infty}(p)-\varepsilon \frac{d P_{\infty}}{d p}+o(\varepsilon)
$$

(see Theorem 6.6). This result is what one would have expected on the basis of the heuristic considerations presented above. It represents the appropriate generalization of our result, mentioned earlier, that $m(\beta)>P_{\infty}(p)$ as $\beta>\infty$ in the dilute ferromagnet.

Thus far, we have only considered the first order effect of antiferromagnetism. Suppose now that $J_{F} \gg J_{A}$ and $p$ is so close to $p_{c}$ that

$$
P_{\infty}(p+\varepsilon) \gg P_{\infty}(p) .
$$

Note that $P_{\infty}(p+\varepsilon)$ is the density of all sites which are ferromagnetically or antiferromagnetically connected to infinity. If, nonperturbatively in $\varepsilon$, the spins on finite ferromagnetic clusters still have a tendency to align, in the majority, in a direction opposite to those of the infinite cluster, and if $p$ and $\varepsilon$ are such that (1.19) holds, then the total magnetization will tend to be opposite to the magnetization of the infinite ferromagnetic cluster (i.e., the direction imposed by the boundary conditions) for $h=0$. We call such a phase a (correlated) random antiferrimagnetic phase. We introduce a simple model of a random ferrite for which we can rigorously prove that the random antiferrimagnetic phase occurs and is stable to perturbations in $p$ and $\varepsilon$. See Sect. 6.C(i). We do not know whether this phase 


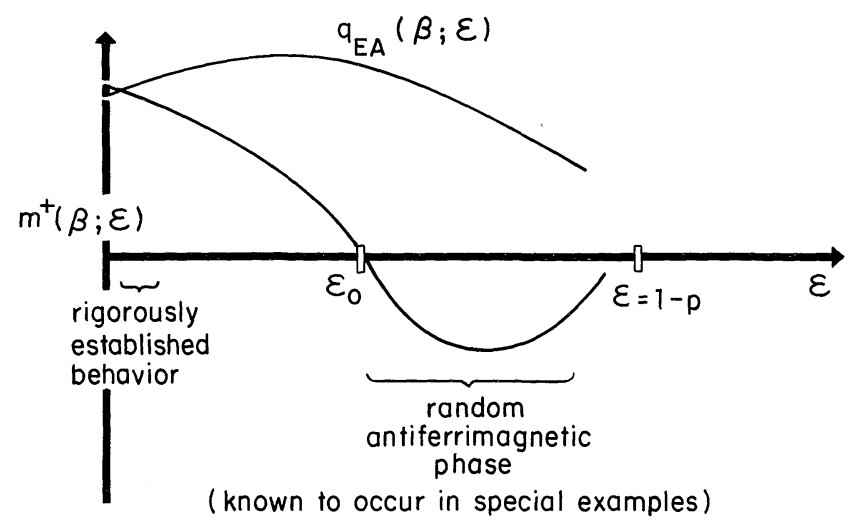

Fig. 2. Behavior of magnetic order parameters in random ferrites

actually occurs in the Bernoulli system or other related random systems. However, if it does occur, our analysis suggests the rather surprising behavior of the spontaneous magnetization illustrated in Fig. 2.

Finally, in Sect. 6.C(ii), we reconsider the Fishman-Aharony map [27] of dilute antiferromagnets in a uniform external field onto a random field ferromagnet. We also speculate on how case $2\left(p<p_{c}, \varepsilon<p_{c}, p+\varepsilon>p_{c}\right)$ may be related to a somewhat frustrated random field Ising model.

\section{Ordering in Predominantly Ferromagnetic Systems}

In this section, we present a simple but general argument which shows that predominantly ferromagnetic Ising systems exhibit a Curie transition. For simplicity, we restrict attention to a system in which (1.3) is a Gaussian distribution of couplings:

$$
d \varrho(J)=(\sqrt{2 \pi} \Delta)^{-1} \exp \left[-(J-\bar{J})^{2} / 2 \Delta^{2}\right] d J .
$$

We show that for $\bar{J}$ sufficiently large and/or $\Delta$ sufficiently small, the system has positive magnetization at low temperature.

The strategy is to use a Peierls estimate on "good" coupling configurations, which are shown to occur with large probability. In subsequent sections, similar estimates will be combined with nonperturbative information on the nature of percolation clusters to obtain sharper results. As usual, the Peierls estimate is performed on contours separating spins of opposite type. In a given configuration, $J=\left\{J_{i j}\right\}$, the energy of a contour $\gamma$ is simply

$$
\mathscr{E}_{J}(\gamma)=2 \sum_{\langle i, j\rangle *} J_{i j}
$$

Here $\langle i, j\rangle^{*}$ denotes the $(d-1)$-cell dual to the bond $\langle i, j\rangle$.

The key ingredient in the analysis is the definition of bad coupling configurations. For some fixed $\delta>0$, we define these by

$$
B=\bigcup_{\gamma: \mathcal{\gamma}_{\ni 0}}\left\{J\left|\mathscr{E}_{J}(\gamma)<2 \delta\right| \gamma \mid\right\}
$$


where the union is over all contours with interior containing the origin. Notice that the complement, $G \equiv B^{c}$, has the property that, for $J \in G$,

$$
\mathscr{E}_{J}(\gamma) \geqq 2 \delta|\gamma|, \quad \text { for every } \gamma \text { with } \quad \bar{\gamma} \ni 0 \text {. }
$$

We first observe that, uniformly in $\beta$, bad configurations are improbable in predominantly ferromagnetic systems:

Proposition 2.1. Provided that $\bar{J}>\delta$,

$$
\operatorname{Prob}(B)<\varepsilon_{1}(\bar{J}, \Delta),
$$

with $\varepsilon_{1}(\bar{J}, \Delta) \searrow 0$ as either $\bar{J} \nearrow \infty$ or $\Delta \searrow 0$.

Proof. By subadditivity of the measure $d R(J)=\prod_{\langle i, j\rangle} d \varrho\left(J_{i j}\right)$, we have

$$
\operatorname{Prob}(B) \leqq \sum_{\gamma: \dot{\gamma} \ni 0} \operatorname{Prob}\left\{J\left|\mathscr{E}_{J}(\gamma)<2 \delta\right| \gamma \mid\right\}
$$

The individual terms in the sum (2.5) may be estimated by noting that $\mathscr{E}_{J}(\gamma)$ is simply a sum of Gaussian random variables, and hence is itself a Gaussian random variable, with mean $2 \bar{J}|\gamma|$ and variance $2 \sqrt{|\gamma|} \Delta$. Thus

$$
\begin{aligned}
\operatorname{Prob}\left\{J\left|\mathscr{E}_{J}(\gamma)<2 \delta\right| \gamma \mid\right\} & \leqq C \exp \left[-[2(\bar{J}-\delta)|\gamma|]^{2} / 4|\gamma| \Delta^{2}\right] \\
& =C \exp \left[-(\bar{J}-\delta)^{2}|\gamma| / \Delta^{2}\right] .
\end{aligned}
$$

The result now follows from a standard Peierls estimate on the contour entropy:

$$
\#\{\gamma|\bar{\gamma} \ni 0,| \gamma \mid=n\} \leqq e^{c_{d} n} .
$$

The previous proposition allows us to focus on good coupling configurations. Next we show that such configurations exhibit ferromagnetic behavior:

Proposition 2.2. Let $\Lambda \subset \mathbb{Z}^{d}$ be any regular finite box, with + boundary conditions. Then, for every $J \in G$,

$$
\left\langle\sigma_{0}\right\rangle_{\beta, \Lambda^{+}}(J) \geqq 1-\varepsilon_{2}(\beta)
$$

with $\varepsilon_{2}(\beta) \downarrow 0$ as $\beta \rtimes \infty$, uniformly in $|\Lambda|$.

Proof. We use the usual Peierls-Griffiths inequality $[4,5]$ :

$$
\left\langle\sigma_{0}\right\rangle_{\beta, \Lambda^{+}}(J) \geqq 1-2 \sum_{\gamma: \Lambda \supset \bar{\gamma} \ni 0} e^{-\beta \mathscr{E}_{J}(\gamma)}
$$

Noting that for all $J \in G, \mathscr{E}_{J}(\gamma)$ obeys the bound (2.4), and using the entropy estimate (2.7), we obtain

$$
\left\langle\sigma_{0}\right\rangle_{\beta, A^{+}}(J) \geqq 1-2 \sum_{n=2 d}^{\infty} e^{-\left(2 \beta \delta-c_{d}\right) n}
$$

In purely ferromagnetic systems (i.e., $J_{i j} \geqq 0, \forall i, j$ ), positivity of the magnetization is an immediate consequence of Propositions 2.1 and 2.2. Indeed, for such systems, the magnetization is given a.s. by [1]

$$
m(\beta)=\lim _{\Lambda \ngtr \mathbb{Z}^{d}}\left\langle\sigma_{0}\right\rangle_{\beta, \Lambda^{+}}(J)=\int\left\langle\sigma_{0}\right\rangle_{\beta,+}(J) d R(J)
$$


The result then follows from decomposing the measure into good and bad configurations, and using the Griffiths-Peierls estimate for $J \in G$.

For systems with antiferromagnetic couplings, the magnetization cannot be related to the expectation value of the spin at the origin, except perhaps as a bound. As usual, the magnetization is defined thermodynamically [see Eq. (1.7)]. Following mutatis mutandis the arguments of van Enter and Griffiths [2], it can be shown that the magnetization so defined is given a.s. by

$$
m(\beta)=\lim _{\Lambda \ngtr \mathbb{Z}^{d}} \max _{\alpha}|\Lambda|^{-1} \sum_{i \in \Lambda}\left\langle\sigma_{i}\right\rangle_{\beta, \Lambda^{\alpha}}(J),
$$

where the maximum is taken over all boundary conditions in a finite volume $\Lambda$, and the limit $\Lambda \rtimes \mathbb{Z}^{d}$ is in the sense of van Hove. In general, the quantity on the right-hand side of (2.11) cannot be expressed in terms of the value of the spin at a given site. Indeed, even in predominantly ferromagnetic systems, there is a nonzero probability that any given site lies in a cluster which is antiferromagnetically connected to the bulk. The physical consequences of this observations will be examined in some detail in Sect. 6.

In order to deal with the above complication, we restrict attention to a finite volume $\Lambda$, and partition the measure $d R(J)$ separately for each $i \in \Lambda$. Thus we define bad configurations with respect to $i, B(i)$, by an expression analogous to (2.3), with the union taken over all contours with interior containing $i$. For each $J \in G(i) \equiv B(i)^{c}$, we have an energy estimate of the form (2.4). It should be clear that the analogues of Propositions 2.1 and 2.2 hold, uniformly in $i$. This enables us to show:

Theorem 2.3. For $\bar{J}$ sufficiently large and/or $\Delta$ sufficiently small, the nearest neighbor Ising magnet with coupling distribution (2.1) exhibits spontaneous magnetization for $\beta$ large enough.

Proof. By (2.11), the magnetization is a.s. bounded below by

$$
m(\beta) \geqq \limsup _{\Lambda \uparrow \mathbb{Z}^{d}}|\Lambda|^{-1} \sum_{i \in \Lambda}\left\langle\sigma_{i}\right\rangle_{\beta, \Lambda^{+}}(J) .
$$

Consider some box $\Lambda$ in the van Hove sequence. By Proposition 2.2, for each $i \in \Lambda$

$$
\left\langle\sigma_{i}\right\rangle_{\beta, \Lambda^{+}}(J) \geqq(-1) \chi_{B(i)}(J)+\left(1-\varepsilon_{2}(\beta)\right) \chi_{G(i)}(J) .
$$

Here we have used $\chi_{E}$ to denote the characteristic function of an event $E$.

By the Birkhoff ergodic theorem, the characteristic functions converge a.s.:

$$
\begin{aligned}
& \lim _{\Lambda \nearrow \mathbb{Z}^{d}}|\Lambda|^{-1} \sum_{i \in \Lambda} \chi_{B(i)}(J)=\operatorname{Prob}(B), \\
& \lim _{\Lambda \nearrow \mathbb{Z}^{d}}|\Lambda|^{-1} \sum_{i \in \Lambda} \chi_{G(i)}(J)=\operatorname{Prob}(G) .
\end{aligned}
$$

The desired result now follows from Proposition 2.1:

$$
m(\beta) \geqq(-1) \varepsilon_{1}(\bar{J}, \Delta)+\left(1-\varepsilon_{2}(\beta)\right)\left(1-\varepsilon_{1}(\bar{J}, \Delta)\right) .
$$

Remark. The argument presented here is not limited to Gaussian distributions. Indeed, these methods can be applied to other coupling distributions provided 
that the mean is large enough and the variance is small enough to allow a bound of the form of Proposition 2.1 on the probability of bad configurations.

\section{Nature of the Infinite Cluster in Percolation}

Although the analysis of the previous section establishes the existence of a Curie transition in dilute magnetic systems, it does not provide optimal results on the amount of dilution which can be tolerated. It turns out that the crucial issue is percolation of ferromagnetism. Indeed, consider a system with a nearest neighbor coupling distribution of the form

$$
d \varrho(J)=[(1-p) \delta(J)+p F(J)] d J,
$$

where $\delta(J)$ is a delta-function at zero coupling, and $F(J)$ is a normalized distribution (without an atom at 0$)$. Then it is easy to show that percolation $\left(P_{\infty}(p)>0\right)$ is a necessary condition for the existence of long range order. In Sect. 4-6, we shall show that percolation of ferromagnetic bonds is also sufficient to achieve ferromagnetic ordering in systems with purely nonnegative couplings in any dimension $^{1}$, and in two-dimensional systems with a limited amount of antiferromagnetism. Our analysis depends on certain properties of infinite clusters, which are reviewed below.

For simplicity, we restrict attention to bond percolation on the hypercubic lattice $\mathbb{Z}^{d}$. It should be noted, however, that the methods and results presented here extend in an obvious fashion to other forms of percolation (e.g., site) and to other lattices. For bond percolation, we regard $\mathbb{Z}^{d}$ as a lattice of sites, and denote by $\mathbb{B}_{d}$ the set of all bonds between nearest neighbor pairs of sites. The bonds $b \in \mathbb{B}_{d}$ are taken to be occupied independently with homogeneous probability $p \in[0,1]$. We denote by $\Omega=\{0,1\}^{\mathbb{B}_{d}}$ the set of configurations of occupied bonds. The Bernoulli measure, $\mu_{p}$, on $\Omega$ is simply the product of the single bond measures at density $p$. The percolation threshold is given by

$$
p_{c}=\inf \left\{p \mid P_{\infty}(p)>0\right\},
$$

where $P_{\infty}(p)$ denotes the $\mu_{p}$-probability that the origin is in an infinite cluster of occupied bonds.

The results we shall need in our subsequent analysis concern the structure of the infinite cluster. According to the approach developed in [14] (see also [28]), whenever $p$ exceeds a threshold value (presumably $p_{c}$ ), it is possible to explicitly construct a subset of the infinite cluster which has the appearance of the original lattice, but occurs with large probability. This may be done by considering block bond events, the probabilities of which approach the unit density fixed point under rescaling. In the remainder of this section, we shall define the block bond events and review their rescaling properties.

In $d=2$, the events we consider are simply crossings of rectangles by occupied bonds. Let $r_{n, L}$ be a rectangle of width $L$ and length $n L$. We denote by $R_{n, L}$ the

1 For $d>2$, this result is proved modulo the usual conjectures of percolation theory [cf. Eq. (3.15)] 
probability of the event that there exists a crossing of $r_{n, L}$ in the long direction by a path of occupied bonds. The following theorem shows that above the percolation threshold, the quantities $R_{n, L}$ tend exponentially to one with $L$.

Theorem 3.1. Whenever $p>p_{c}$, there exists a $\xi(n, p)<\infty$ such that

$$
R_{n, L} \geqq 1-e^{-L / \xi(n, p)} .
$$

Proof. It is a fundamental result of two-dimensional percolation theory [29, 30] (see also [31]) that whenever $p>p_{c}, \lim _{L \rightarrow \infty} R_{n, L}=1$. On the other hand, it can be shown $[14,28]$ that there exists a constant $c(n)$ such that

$$
R_{n, L} \geqq 1-c(n) e^{-1} \Rightarrow R_{n, 2^{l} L} \geqq 1-c(n) e^{-2^{l}},
$$

which establishes that the convergence is exponential.

Remark. Although (3.3) is sufficient for our purposes, it is not difficult to establish the stronger result that

$$
\lim _{L \rightarrow \infty}\left[-\frac{1}{L} \log \left(1-R_{n, L}\right)\right]=\xi^{-1}(p)
$$

exists and is independent of $n$. Indeed, it can be shown that $\xi(p)$ is precisely the correlation length of the system, which is here defined as the inverse of the decay rate of the dual connectivity function. That is, if $\tau_{0 x}^{*}$ is the probability that the points 0 and $x$ are connected by a path of dual bonds, then

$$
\xi^{-1}(p) \equiv \lim _{x \rightarrow \infty}\left[-\frac{1}{x} \log \tau_{0 x}^{*}\right] .
$$

That the above limit exists has been known for some time [32, 33] (see also [31]).

With regards to the claim (3.5), we note that if $L_{0}(p)$ is defined to be the smallest length such that the rescaling hypothesis (3.4) is satisfied, i.e.,

$$
L_{0}(p)=\inf \left\{L \mid R_{n, L}(p) \geqq 1-c(n) e^{-1}\right\},
$$

then we have:

Proposition 3.2. Up to logarithms, $L_{0}(p)$ coincides with $\xi(p)$.

Proof. For definiteness, we take $n=2$ and use $c(2)=1 / 16$. First observe that if we surround the origin with an annulus consisting of four translations (and rotations) of $r_{2, L}$, and if crossings occur in all of them, then there is no path of dual bonds from $(0,0)$ to $(0, L)$. Thus

$$
\tau_{0 L}^{*} \leqq 1-R_{2, L}^{4} .
$$

If we now take $L=2^{l} L_{0}$ and use (3.4) this implies

$$
\tau_{0 L}^{*} \leqq e^{-L / L_{0}},
$$

which, by the existence of $\xi^{-1}(p)$, implies $\xi^{-1}(p) \geqq L_{0}^{-1}(p)$.

On the other hand, suppose we have $L_{0}>1$ satisfying (3.7). Denote by $S_{1}$ and $S_{2}$ the two long faces of the rectangle $r_{2, L_{0}-1}$. By subadditivity

$$
1-R_{2, L_{0}-1} \leqq \sum_{\substack{x \in S_{1} \\ y \in S_{2}}} \tau_{x y}^{*} .
$$


Using the (easily verified) fact that $\tau_{x y}^{*} \leqq e^{-\left(L_{0}-1\right) / \xi}$, and the rescaling hypothesis, we have

$$
c e^{-1} \leqq 1-R_{2, L_{0}-1} \leqq 4 L_{0}^{2} e^{-\left(L_{0}-1\right) / \xi},
$$

which implies

$$
\xi^{-1}(p) \leqq\left[c_{1} \log L_{0}(p)+c_{2}\right] / L_{0}(p)
$$

for some explicitly computable $c_{1}, c_{2}$.

The quantities $R_{n, L}$ will enable us to construct, with large probability, configurations which guarantee ferromagnetic ordering in two dimensions. However, such a construction relies on the obvious intersection property of bonds in $d=2$, namely that overlapping paths of bonds necessarily intersect. In higher dimensions, intersection of rescaled bond events is not guaranteed by purely geometrical considerations. For $d=3$, this difficulty was overcome in [14] by formulating conditions which ensure uniqueness of the infinite cluster, and then using the uniqueness to "force" intersection of bond events on sufficiently large length scales. We shall invoke such a procedure, as reviewed below, for all $d \geqq 3$.

Consider a partition of the lattice $\mathbb{Z}^{d}$ into layers of finite thickness $k$ in all but two dimensions: $\mathbb{Z}^{d} \times\{0, \ldots, k\}^{d-2}$. Let $\hat{P}_{\infty}^{k}(p)$ denote the probability that the origin is part of an infinite cluster of occupied bonds which lies entirely within a given quadrant of the $k$-layer. This defines a quadrant percolation threshold for the $k$-layer:

$$
\hat{p}_{c}^{k}=\inf \left\{p \mid \hat{P}_{\infty}^{k}(p)>0\right\} .
$$

Since the thresholds $\hat{p}_{c}^{k}$ are monotone decreasing in $k$, we may define

$$
\hat{p}_{c}^{\infty}=\lim _{k \rightarrow \infty} \hat{p}_{c}^{k} .
$$

Indeed, it is natural to conjecture that the limit of the quadrant layer thresholds coincides with the percolation threshold for the full $d$-dimensional lattice ${ }^{2}$ :

$$
\hat{p}_{c}^{\infty}=p_{c} .
$$

Our results on ferromagnetic ordering for $d \geqq 3$ will be proved modulo this conjecture, i.e. for $p>\hat{p}_{c}^{\infty}$.

The strategy is to note that if $p>\hat{p}_{c}^{\infty}$, then there is some finite $k$ such that $p>\hat{p}_{c}^{k}$. Thus we may deal exclusively with the $k$-layer, $\mathbb{Z}^{2} \times\{0, \ldots, k\}^{d-2}$. The block bonds can now be constructed using a coarse-graining argument. Consider a partition of the $k$-layer, $\mathbb{Z}^{2} \times\{0, \ldots, k\}^{d-2}$, into $L \times L \times k^{d-2}$ hypercubes oriented at $45^{\circ}$ with respect to the $\mathbb{Z}^{2}$ axes. At the center of each hypercube, construct a $l \times l \times k^{d-2}$ hypercube $(l<L)$, oriented in the same fashion. The larger boxes will be denoted by $C_{L}(i)$ and the smaller by $C_{l}(i)$, where $i$ indicates a point on the coarse-grained, rotated lattice. Roughly speaking, the smaller boxes, $C_{l}$, should be regarded as

2 One can define an analogous critical point, $p_{c}^{\infty}$, as the limit of full (i.e., nonquadrant) $k$-layer thresholds. It is, in fact, possible to show [34] that $\hat{p}_{c}^{\infty}=p_{c}^{\infty}$, thereby reducing the conjecture (3.15) to $p_{c}^{\infty}=p_{c}$. The analogue of this latter conjecture for Ising systems has recently been proved by Aizenman [35] 
sites, and nearest neighbor pairs of larger boxes, $C_{L}(i) \cup C_{L}(j)$, should be viewed as bonds of the coarse-grained lattice. Block bonds may be constructed from the following two events:

(1) Let $i$ and $j$ denote a pair of nearest neighbor sites (separated by a Euclidean distance $\sqrt{2} L)$. The bond connection event, $b_{\langle i, j\rangle}$, is defined to be the occurrence of a path of occupied bonds connecting $\partial C_{l}(i)$ to $\partial C_{l}(j)$ which lies entirely within $C_{L}(i) \cup C_{L}(j)$

(2) Let $i$ denote a site on the coarse-grained lattice. The site uniqueness event, $\sigma_{i}$, is the event that all paths of occupied bonds which connect $\partial C_{l}(i)$ to $\partial C_{L}(i)$ are connected to each other within $C_{L}(i)$. That such events occur with large probability is expressed in the following:

Theorem 3.3 [14, Propositions 4.6 and 4.7]. Suppose $p>\hat{p}_{c}^{k}$. Then

(1) for all $\varepsilon>0$, there exists an l large enough so that

$$
\operatorname{Prob}\left(b_{\langle i, j\rangle}\right) \geqq 1-\varepsilon
$$

uniformly in $L$, provided that $L>l$.

(2) For any fixed $l$, for all $\varepsilon>0$, there exists an L large enough so that

$$
\operatorname{Prob}\left(\Im_{i}\right) \geqq 1-\varepsilon \text {. }
$$

Using Theorem 3.3, we may now construct block bonds of arbitrarily high density by "pasting together" bond connection events for pairs of sites with uniqueness events at the vertices. This will be the strategy followed in Sect. 5.

\section{Ferromagnetic Ordering in Two Dimensions}

In the next two sections, we consider a dilute ferromagnet with a coupling distribution of the form (3.1), where $F(J)$ is any nonnegative distribution (without an atom at 0 ). Modulo the usual conjectures of percolation theory, it is shown that wherever $p$ exceeds the percolation threshold, the system exhibits spontaneous magnetization at sufficiently low temperature.

In order to establish this result, it suffices to consider a system with Bernoulli coupling distribution

$$
d \varrho_{p}(J)=\left[(1-p) \delta(J)+p \delta\left(J-J_{F}\right)\right] d J,
$$

where $J_{F}>0$ is the ferromagnetic bond strength. Indeed, given a system of the form (3.1) with $p>p_{c}$, it is possible to define a coupling strength $\widetilde{J}_{F}(\lambda)>0$ by

$$
\int_{0}^{\tilde{J}_{F}^{(\lambda)}} F(J) d J=\lambda\left(p-p_{c}\right)
$$

for some ${ }^{3} \lambda \in(0,1)$. Bonds of strength exceeding $\tilde{J}_{F}(\lambda)$ occur with density $\tilde{p}(\lambda)$ $=p_{c}+(1-\lambda)\left(p-p_{c}\right)>p_{c}$. By the GKS inequality [36], the magnetization of the original system (3.1) is bounded below by that of the Bernoulli system with bond

3 If $F(J)$ is smooth and has support for all $J>0$, we may choose any $\lambda \in(0,1)$. If $F(J)$ vanishes on some open set and/or contains delta functions at positive couplings, $\lambda$ must be adjusted accordingly to obtain a well-defined value of $\widetilde{J}_{F}(\lambda)$ 
strength $\tilde{J}_{F}(\lambda)$ and density $\tilde{p}(\lambda)$. In fact, in two dimensions, the more general result follows without the use of correlation inequalities by simply replacing our energy estimates on the Bernoulli system for bonds of strength $J_{F}$ and density $p$ by estimates for bonds of strength exceeding $\tilde{J}_{F}(\lambda)$ at density $\tilde{p}(\lambda)$.

For the two-dimensional system, existence of ferromagnetic ordering for $p>p_{c}$ was first established by Georgii [13]. In this section, we offer a simple proof of the two-dimensional result which has the additional advantage that it makes no use of correlation inequalities. This will enable us to prove that ordering persists in the presence of some antiferromagnetic impurities (see Sect. 6).

Our argument is again a Peierls estimate on good coupling configurations; however, good is now defined with respect to a coarse-grained lattice. To this end, we divide $\mathbb{Z}^{2}$ into $2 L \times 2 L$ boxes and denote the resulting lattice by $2 L \mathbb{Z}^{2}$. The boxes may be grouped into four sublattices, $\mathbb{L}_{\mathrm{I}}, \ldots, \mathbb{L}_{\mathrm{IV}}$, each sublattice consisting of boxes which have no sides in common (see Fig. 3a). The annulus of width $L$ surrounding a given box $C_{\mathbf{x}}, \mathbf{x} \in 2 L \mathbb{L}^{2}$, will be called the boundary region, $\partial C_{\mathbf{x}}$, of the box $C_{\mathbf{x}}$. Although the boundary regions of different sublattices overlap, the boxes and boundary regions of a given sublattice form a disjoint partition of $\mathbb{Z}^{2}$ (see Fig. 3b).

A box, $C_{\mathbf{x}}$, is said to be ferromagnetically sealed if there is a circuit of ferromagnetic bonds surrounding $C_{\mathbf{x}}$ which lies entirely in $\partial C_{\mathbf{x}}$. If a box is not sealed, it is said to leak. It is easy to see that the sealing events

$$
\mathscr{S}_{\mathbf{x}}=\left\{J \mid C_{\mathbf{x}} \text { is ferromagnetically sealed }\right\}
$$

have probability independent of $\mathbf{x}$, and are statistically independent for each pair $\mathbf{x}, \mathbf{y}$ belonging to the same sublattice.

When the density of ferromagnetic bonds exceeds the percolation threshold, the probability of a leak tends to zero exponentially with the scale of the coarsegraining:
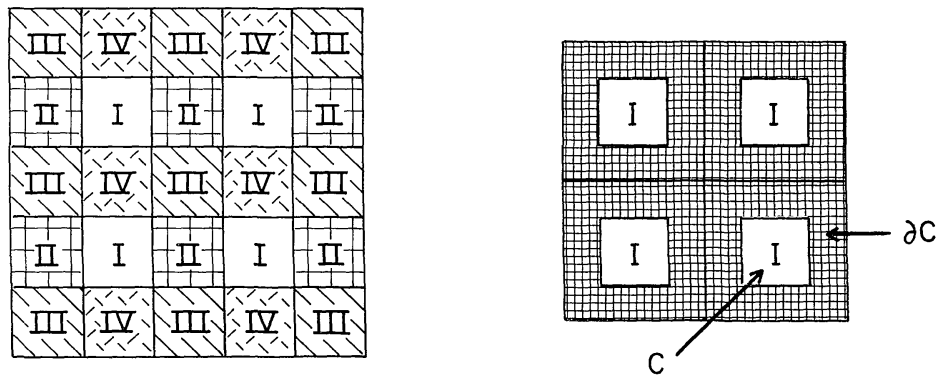

Partition of $\mathbb{Z}^{2}$

Fig. 3a and b. Partition of $\mathbb{Z}^{2}$

Proposition 4.1. Suppose $p>p_{c}$ and define

$$
P_{\text {leak }}(p, L) \equiv 1-\operatorname{Prob}_{p}\left(\mathscr{S}_{\mathbf{0}}\right) .
$$

Then $P_{\text {leak }}(p, L)$ may be made arbitrarily small by taking $L$ sufficiently large. Indeed,

$$
P_{\text {leak }}(p, L) \leqq(\text { const }) e^{-L / \xi(4, p)} .
$$


Proof. By subadditivity of the measure,

$$
P_{\text {leak }}(p, L) \leqq 4\left(1-R_{4, L}\right),
$$

where $R_{4, L}$ is the probability of crossing a $4 L \times L$ rectangle. The result is an immediate consequence of Theorem 3.1 and Proposition 3.2.

Remark. The length $\xi(p)$ will determine the scale on which we must coarse-grain the lattice. It turns out (see Sect. 6) that this scale also determines how much antiferromagnetism the system can tolerate. Since we may identify $\xi(p)$ as the correlation length of the associated percolation system (see Remark following Theorem 3.1), this implies that the critical antiferromagnetic tolerance is related to the divergence (presumably a power law) of the correlation length as $p \searrow p_{c}$.

We are now in a position to outline the basic strategy. Any Peierls contour can be classified according to the boxes that it visits. (This amounts to a coarsegraining of the contours.) If, with large probability, a given fraction of these boxes are sealed, the contour typically has an energy which scales with its length. This drives a Curie transition at sufficiently large $\beta$.

Let us introduce the relevant notations and definitions. An arrangement is a finite, connected collection of boxes which surrounds or contains the origin. The set $\Gamma$ of all arrangements may be partitioned into four disjoint subsets: $\Gamma_{\mathrm{I}} \subset \Gamma$ are those arrangements containing at least as many boxes of $\mathbb{L}_{\mathbf{I}}$ as of any other sublattice; $\Gamma_{\mathrm{II}} \subset \Gamma \backslash \Gamma_{\mathrm{I}}$ are arrangements containing a plurality of boxes of $\mathbb{L}_{\mathrm{II}}$, and so on.

An arrangement is said to be bad if more than (say) half of the boxes of the dominant sublattice leak. A configuration, $J$, is said to be bad if it has any bad arrangements of boxes. We denote the set of all bad configurations by $B_{L}$ and its complement by $G_{L}$.

Proposition 4.2. Suppose $p>p_{c}$. Then $\operatorname{Prob}_{p}\left(B_{L}\right)$ may be made arbitrarily small, by taking $L$ sufficient large.

Proof. A given arrangement with $M$ boxes on the dominant sublattice is bad with probability

$$
\sum_{N>M / 2}\left(\begin{array}{c}
M \\
N
\end{array}\right) P_{\text {leak }}^{N}\left(1-P_{\text {leak }}\right)^{M-N} \leqq 2^{M} P_{\text {leak }}^{M / 2} .
$$

The number of such arrangements is bounded above by the number of arrangements of not more than $4 M$ boxes. This in turn is bounded above by $e^{4 a_{2} M}$, where $a_{2}<\infty$ can be related to the two-dimensional polymer constant. Thus

$$
\operatorname{Prob}_{p}\left(B_{L}\right) \leqq \sum_{M=1}^{\infty} e^{4 a_{2} M} 2^{M} P_{\text {leak }}^{M / 2} .
$$

The desired result now follows from Proposition 4.1.

We are now ready to establish the existence of a ferromagnetic transition:

Theorem 4.3. Suppose $p>p_{c}$. Then the nearest neighbor Ising magnet with coupling distribution (4.1) exhibits spontaneous magnetization for $\beta$ large enough. Moreover,

$$
m(\beta) \nrightarrow P_{\infty}(p) \text { as } \beta \uparrow \infty .
$$


Proof. Consider the restriction of the system to a finite box, $\Lambda \subset 2 L \mathbb{Z}^{2}$, with + boundary conditions. Let $\mathcal{O}_{A}$ denote the event that the origin is in the ferromagnetic connected component of the boundary, $\partial \Lambda$. Defining $d \mu_{p}=\prod_{\langle i, j\rangle} d \varrho_{p}\left(J_{i j}\right)$, the magnetization is given a.s. by

$$
\begin{aligned}
m(\beta) & =\lim _{\Lambda \uparrow \mathbb{Z}^{2}} \int\left\langle\sigma_{0}\right\rangle_{\beta, \Lambda^{+}}(J) d \mu_{p}(J) \\
& =\lim _{\Lambda \ngtr \mathbb{Z}^{2}} \operatorname{Prob}_{p}\left(\mathcal{O}_{\Lambda}\right) \int\left\langle\sigma_{0}\right\rangle_{\beta, \Lambda^{+}}(J) d \mu_{p}\left(J \mid \mathcal{O}_{\Lambda}\right),
\end{aligned}
$$

since $\left\langle\sigma_{0}\right\rangle_{\beta, \Lambda^{+}}(J)$ vanishes for those configurations in which the origin is disconnected from $\partial \Lambda$. Defining $\mathcal{O}_{\infty}$ as the event that the origin is in the infinite cluster, we have

$$
\lim _{\Lambda>\mathbb{Z}^{2}} \operatorname{Prob}_{p}\left(\mathcal{O}_{\Lambda}\right)=\operatorname{Prob}_{p}\left(\mathcal{O}_{\infty}\right) \equiv P_{\infty}(p)
$$

by continuity of the measures. Thus it suffices to show that the integral on the right-hand side of (4.7) tends to 1 as $\beta \uparrow \infty$, uniformly in $|\Lambda|$.

To this end, we first decompose the measure into good and bad $^{4}$ configurations:

$$
\begin{aligned}
& \int_{J \in \mathscr{O}_{A}}\left\langle\sigma_{0}\right\rangle_{\beta, \Lambda^{+}}(J) d \mu_{p}(J) \\
& \geqq(-1) \operatorname{Prob}_{p}\left(B_{L} \cap \mathcal{O}_{\Lambda}\right)+\int_{J \in G_{L} \cap \mathcal{O}_{A}}\left\langle\sigma_{0}\right\rangle_{\beta, \Lambda^{+}}(J) d \mu_{p}(J) .
\end{aligned}
$$

Now note that $B_{L}$ is a negative event, while $G_{L}$ and $\mathcal{O}_{A}$ are positive. Thus by the FKG inequality [37] for percolation (first proved by Harris [38]), we have

$$
\begin{aligned}
& \int\left\langle\sigma_{0}\right\rangle_{\beta, \Lambda^{+}}(J) d \mu_{p}\left(J \mid \mathcal{O}_{\Lambda}\right) \\
& \quad \geqq(-1) \operatorname{Prob}_{p}\left(B_{L}\right)+\left\{\int\left\langle\sigma_{0}\right\rangle_{\beta, \Lambda^{+}}(J) d \mu_{p}\left(J \mid G_{L} \cap \mathcal{O}_{\Lambda}\right)\right\} \operatorname{Prob}_{p}\left(G_{L}\right) .
\end{aligned}
$$

Next, we must obtain a lower bound on $\left\{\left\langle\sigma_{0}\right\rangle_{\beta, A^{+}}(J) \mid J \in G_{L} \cap \mathcal{O}_{\infty}\right\}$. This is done by again invoking the Peierls-Griffiths inequality $[4,5]$ :

$$
\left\langle\sigma_{0}\right\rangle_{\beta, A^{+}}(J) \geqq 1-2\left\langle\bigcup_{\gamma: \bar{\gamma} \ni 0} A_{\gamma}\right\rangle_{\beta, A^{+}}(J),
$$

where $A_{\gamma}$ denotes the event that $\gamma$ is the outermost contour surrounding the origin which separates spins of opposite type. Here, however, our estimate on $\left\langle\cup A_{\gamma}\right\rangle$ will be performed on coarse-grained contours.

Each contour $\gamma$ can be classified according to the boxes that it visits. Recall that $\Gamma_{K}$ denotes those arrangements which have a plurality of boxes on sublattice $\mathbb{L}_{K}$. We partition each $\Gamma_{K}$ on the basis of the number of boxes of the dominant sublattice, denoting by $\Gamma_{\mathrm{K}}^{M}$ those arrangements in $\Gamma_{K}$ which contain $M$ boxes of sublattice $K$. Then the set of all arrangements is given by the disjoint partition

$$
\Gamma=\bigcup_{K=I}^{I V} \bigcup_{M=1}^{\infty} \Gamma_{K}^{M} \text {. }
$$

4 Since this system has purely nonnegative couplings, correlation inequalities allow us to bound $\left\langle\sigma_{0}\right\rangle_{\beta, \Lambda^{+}}\left(J \in B_{L}\right)$ below by zero, rather than by $(-1)$. We use the less efficient bound in order to facilitate the introduction of antiferromagnetic bonds (see Sect. 6) 
A contour $\gamma$ is said to be in $\Gamma_{K}^{M}$, denoted by $\gamma \in \Gamma_{K}^{M}$, if the arrangement of boxes that it visits is in $\Gamma_{K}^{M}$.

In order to estimate $\left\langle\cup A_{\gamma}\right\rangle$, we first use subadditivity:

$$
\left\langle\bigcup_{\gamma: \bar{\gamma} \ni 0} A_{\gamma}\right\rangle_{\beta, \Lambda^{+}}(J) \leqq \sum_{K, M}\left\langle\bigcup_{\gamma \in \Gamma_{K}^{M}} A_{\gamma}\right\rangle_{\beta, \Lambda^{+}}(J) \text {. }
$$

If $J \in G_{L}$, then every contour $\gamma \in \Gamma_{K}^{M}$ must visit at least $(1 / 2) M$ sealed boxes. Unless $M=1$, this means that $\gamma$ must cross at least $2(1 / 2) M=M$ ferromagnetic bonds. If, in addition, $J \in \mathcal{O}_{\Lambda}$, then the same result holds for $M=1$, since $\gamma$ must cross the connection of the origin to $\partial \Lambda$. This provides the energy estimate

$$
\mathscr{E}_{J}(\gamma) \geqq 2 \beta J_{F} M \quad \forall J \in G_{L} \cap \mathcal{O}_{\Lambda} \quad \text { and } \quad \forall \gamma \in \Gamma_{K}^{M} .
$$

The size of $\Gamma_{K}^{M}$ can be bounded above by the number of contours encircling the origin of length smaller than $(4 M)(2)(2 L)^{2}$. Thus

$$
\#\left\{\gamma \mid \gamma \in \Gamma_{K}^{M}\right\} \leqq(\text { const }) \sum_{n \leqq 32 M L^{2}} e^{c_{2} n} \leqq e^{\bar{c} M L^{2}}
$$

for some $\bar{c}<\infty$. By (4.12)-(4.14), we have

$$
\left\langle\bigcup_{\gamma: \bar{\gamma} \ni 0} A_{\gamma}\right\rangle_{\beta, \Lambda^{+}}\left(J \in G_{L} \cap \mathcal{O}_{\Lambda}\right) \leqq 4 \sum_{M=1}^{\infty} e^{-2 \beta J_{F} M} e^{+\bar{c} M L^{2}},
$$

uniformly in $|\Lambda|$.

Thus

$$
\begin{aligned}
& \int\left\langle\sigma_{0}\right\rangle_{\beta,+}(J) d \mu_{p}\left(J \mid \mathcal{O}_{\infty}\right) \\
& \quad \geqq-\operatorname{Prob}\left(B_{L}\right)+\operatorname{Prob}\left(G_{L}\right)\left[1-8 \sum_{M=1}^{\infty} e^{-\left(2 \beta J_{F}-\bar{c} L^{2}\right) M}\right] .
\end{aligned}
$$

By Proposition 4.2, the lower bound can be made positive by first choosing $L$ then $\beta$ sufficiently large. Indeed, as $L \rightarrow \infty$ and $\beta \rightarrow \infty$, the bound tends to 1 .

Remark. For future reference, we note that $\operatorname{Prob}\left(G_{L}\right)>1 / 2$ is a sufficient condition for positivity of the lower bound (4.16), provided that $\beta$ is large enough. By Proposition 4.1 and Eq. (4.6) this in turn implies that we need only coarse-grain up to the scale of the correlation length to establish spontaneous magnetization.

Corollary. For $p>p_{c}$ and $\beta$ sufficiently large, the infinite cluster is almost surely magnetized with constant magnetization $m(\beta) / P_{\infty}(p)$.

Proof. The overall magnetization is given a.s. by

$$
m(\beta)=\lim _{\Lambda \ngtr \mathbb{Z}^{2}} \frac{1}{|\Lambda|} \sum_{i \in \Lambda}\left\langle\sigma_{i}\right\rangle_{\beta, \Lambda^{+}}(J) .
$$

Defining $\mathscr{C}_{\infty}(J)$ to be the infinite cluster in configuration $J$, this may be rewritten as

$$
\lim _{\Lambda \rtimes \mathbb{Z}^{2}}\left\{\frac{\left|\Lambda \cap \mathscr{C}_{\infty}(J)\right|}{|\Lambda|} \frac{1}{\left|\Lambda \cap \mathscr{C}_{\infty}(J)\right|} \sum_{i \in \Lambda \cap \mathscr{C}_{\infty}(J)}\left\langle\sigma_{i}\right\rangle_{\beta, \Lambda^{+}}(J)+\frac{1}{|\Lambda|} \sum_{i \in \Lambda \mid \mathscr{C}_{\infty}(J)}\left\langle\sigma_{i}\right\rangle_{\beta, \Lambda^{+}}(J)\right\} .
$$


We first argue that the second term above vanishes as $\Lambda \nearrow \mathbb{Z}^{2}$. Indeed, it consists of spins in finite clusters which make a nonzero contribution only if the cluster is connected to $\partial \Lambda$. However, these boundary effects vanish in the infinite volume limit.

By the ergodic theorem, the factor $\left|\Lambda \cap \mathscr{C}_{\infty}(J)\right| /|\Lambda|$ tends a.s. to $P_{\infty}(p)$ as $\Lambda \rtimes \mathbb{Z}^{2}$. Evidently

$$
\lim _{\Lambda \nearrow \mathbb{Z}^{2}} \frac{1}{\left|\Lambda \cap \mathscr{C}_{\infty}(J)\right|} \sum_{i \in \Lambda \cap \mathscr{C}_{\infty}(J)}\left\langle\sigma_{i}\right\rangle_{\beta, \Lambda^{+}}(J)
$$

is a.s. constant $\left(=m(\beta) / P_{\infty}(p)\right)$.

Remark. 1. The corollary immediately implies the a.s. existence of at least two Gibbs states in this system. Indeed, it provides a convenient and intuitive characterization of the Gibbs states in terms of the (+ or -$)$ magnetization of the infinite cluster.

2. Although the overall magnetization is the appropriate order parameter for this system, the magnetization of the infinite cluster will turn out to be the relevant quantity for analysis of systems with some antiferromagnetic couplings. Indeed, since the infinite cluster contains a finite fraction $\left(=P_{\infty}(p)\right)$ of the spins in the system, its magnetization always implies positivity of the Edwards-Anderson order parameter $q_{\mathrm{EA}}$.

\section{Ferromagnetic Ordering in $d \geqq 3$}

We now extend the results of the previous section to dilute ferromagnets in $d \geqq 3$. It is shown that whenever $p>\hat{p}_{c}^{\infty}$, the Bernoulli system exhibits spontaneous magnetization for $\beta$ sufficiently large.

Existence of ferromagnetic order above the percolation threshold is a delicate question in more than two dimensions. Indeed, a naive extension of the methods of the previous section does not apply. This is a consequence of two related properties of the percolation system in $d \geqq 3$ :

1. Overlapping paths of bonds need not intersect.

2. For $p \gtrsim p_{c}$, the $(d-1)$-cells dual to vacant bonds can form infinite clusters. Of particular concern is the formation of infinite "tubes" of $(d-1)$-cells which do not interfere with the infinite bond cluster. A sufficient condition to prevent $(w \cdot p \cdot 1)$ the existence of such objects is $p>1-\pi_{c}^{*}$, where $\pi_{c}^{*}$ is the threshold at which the expected size of $(d-1)$-cell clusters diverges.

In $d=2$, it is known that $p_{c}=1-\pi_{c}^{*}[29,30]$. This absence of percolation of dual objects was crucial for the analysis of the previous section. There, the infinite cluster formed a mesh which partitioned the lattice into finite regions on the scale of the correlation length. As a consequence, the intersection of a dual contour with the infinite cluster was, with large probability, a connected set with size proportional to the size of the contour. It was this property which provided the necessary energy estimates to drive the Peierls-Griffiths argument.

In $d \geqq 3$, it is generally the case that $1-\pi_{c}^{*}>p_{c}$. As one might expect, for $p>1$ $-\pi_{c}^{*}$, it is possible to apply the arguments of the previous section and indeed, 


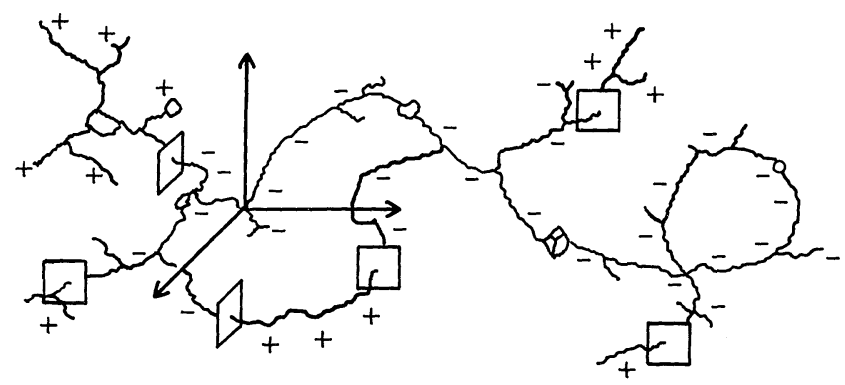

Fig. 4. A Peierls contour (plaquettes) of the 3-dimensional percolation cluster
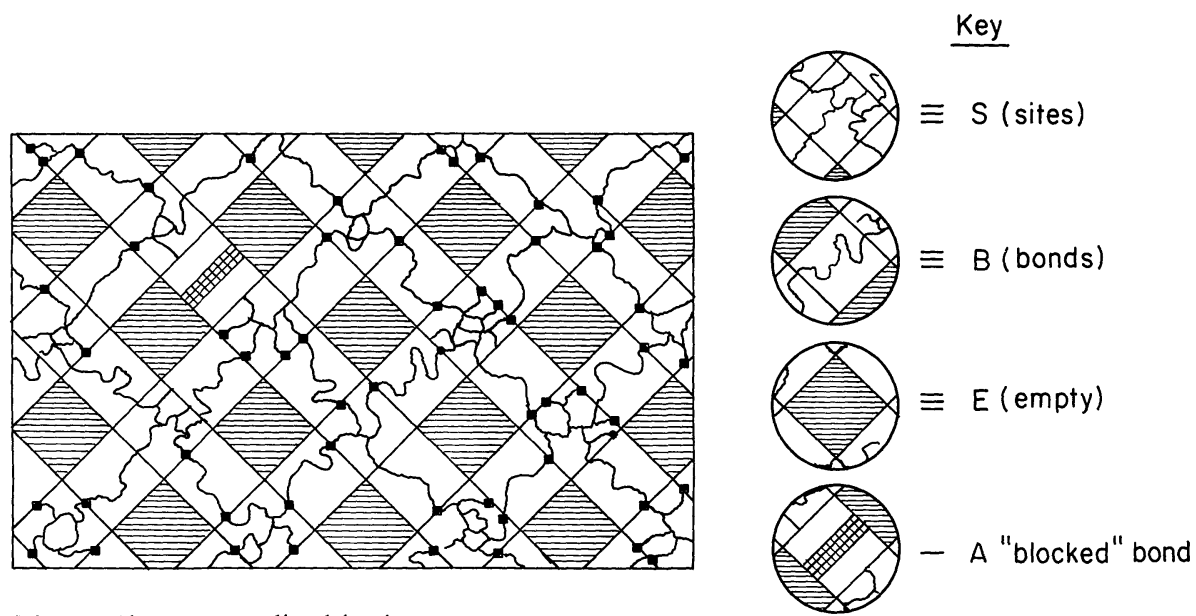

Fig. 5. The renormalized lattice

even to extend the results obtained in Sect. 6. Similar conditions have been exploited in the analysis of other random media problems $[39,40]$. However, when $p_{c}<p<1-\pi_{c}^{*}$, paths can dodge the infinite cluster indefinitely. Thus the intersection of a dual surface with the infinite cluster need not form a connected set, no matter how we coarse-grain the lattice (see Fig. 4).

In systems with purely nonnegative couplings we may, however, invoke the GKS correlation inequalities [36]. For our purposes, this translates into the proposition that if we can establish spontaneous magnetization utilizing only a subset of the infinite cluster, then there is magnetization in the full system. Thus we will assume that $p>\hat{p}_{c}^{\infty}$, which implies that $p>\hat{p}_{c}^{k}$ for some finite $k$, and show that (a subset of) the infinite cluster in a $k$-layer has nonzero magnetization for $\beta$ sufficiently large.

The scheme is to retain only a portion of the (unique!) infinite cluster which has the connectedness properties of $\mathbb{Z}^{2}$. To do this, we use a modification of the construction outlined in Sect. 3. First, the $k$-layer $\mathbb{Z}^{2} \times\{0, \ldots, k\}^{d-2}$ is partitioned into $L \times L \times k^{d-2}$ hypercubes oriented at $45^{\circ}$ with respect to the $\mathbb{Z}^{2}$ axes. The cubes are then grouped into three sublattices, $\mathbb{E}, \mathbb{S}$, and $\mathbb{B}$, as shown in Fig. 5. We shall 
regard the cubes of $\mathbb{S}$ and $\mathbb{B}$ as the sites and bonds, respectively, of the coarsegrained lattice. Thus we denote by $C_{i}, i \in 2 L \mathbb{Z}^{2}$, a cube of sublattice $\mathbb{S}$, and by $C_{i j}$ that cube of sublattice $\mathbb{B}$ between the nearest neighbor pair $C_{i}, C_{j} \in \mathbb{S}$.

The ferromagnetic bonds discarded from a particular cube will be determined by the sublattice to which the cube belongs. From cubes of sublattices $\mathbb{E}$, we shall delete all ferromagnetic material. If $C_{i} \in \mathbb{S}$, we shall retain only a subset, $S\left(C_{i}\right)$, of ferromagnetic bonds which satisfies the following two conditions:

1) Each $b \in S\left(C_{i}\right)$ is connected by a path of ferromagnetic bonds to all four walls of $\partial C_{i}$.

2) Each pair $b, b^{\prime} \in S\left(C_{i}\right)$ is connected by a path of ferromagnetic bonds within $C_{i}$.

The ferromagnetism we shall retain in $C_{i j} \in \mathbb{B}$ is that subset, $B\left(C_{i j}\right)$, which constitutes the shortest crossings of ferromagnetic bonds from the set $S\left(C_{i}\right)$ to the set $S\left(C_{j}\right)$. [If either $S\left(C_{i}\right)$ or $S\left(C_{j}\right)$ is empty, or if there is no such crossing, delete all ferromagnetism. If there are several such sets, it is easy to devise prescriptions for choosing a single one.]

It should be clear that the events $S\left(C_{i}\right) \neq \emptyset$ and $B\left(C_{i j}\right) \neq \emptyset$ are easily constructed from the site uniqueness event, $s_{i}$, and bond connection event, $b_{\langle i, j\rangle}$, discussed in Sect. 3. It then follows immediately from Theorem 3.3 that the block bond event

$$
\mathscr{B}_{i j}=\left\{J \mid S\left(C_{i}\right) \neq \emptyset \text { and } B\left(C_{i j}\right) \neq \emptyset \text { and } S\left(C_{j}\right) \neq \emptyset\right\}
$$

occurs with arbitrarily large density:

Proposition 5.1. Suppose $p>\hat{p}_{c}^{k}$. Then, for any $\varepsilon>0$, there is an $L$ so large that $\operatorname{Prob}_{p}\left(\mathscr{B}_{i j}\right)>1-\varepsilon$.

The scheme of the proof is now straightforward. We shall deal exclusively with the coarse-grained lattice, and define "good" configurations in terms of the block bond events. Then, provided that we delete from any configuration, $J$, those ferromagnetic bonds which are not necessary for $J$ to be "good," it will be possible to do a Peierls-Griffiths estimate similar to those done previously.

Let $\mathbb{V}$ denote the coarse-grained, rotated lattice. The dual lattice, $\mathbb{V}^{*}$, has as "sites" the cubes of the sublattice $\mathbb{E}$. A contour $\Gamma$ on $\mathbb{V} *$ is sait to be good if the event $\mathscr{B}_{i j}$ occurs for more than (say) half of the bonds $\langle i, j\rangle \in \mathbb{V}$ which $\Gamma$ crosses. We denote by $G_{L}$ those configurations in which every contour surrounding the origin is good. By proof along the lines of Proposition 4.2, we can show:

Proposition 5.2. Suppose $p>\hat{p}_{c}^{k}$. Then $\operatorname{Prob}_{p}\left(G_{L}\right)$ may be made arbitrarily close to 1 by taking $L$ sufficiently large.

Our principal result is the following:

Theorem 5.3. Suppose $p>\hat{p}_{c}^{\infty}$. Then for $\beta$ sufficiently large, the nearest neighbor Ising system with Bernoulli couplings exhibits spontaneous magnetization.

Proof. Let $k$ be the smallest integer such that $p>\hat{p}_{c}^{k}$. Consider a partition of the $k$-layer $\mathbb{Z}^{2} \times\{0, \ldots, k\}^{d-2}$ into cubes of scale $L$ large enough so that $\operatorname{Prob}_{p}\left(G_{L}\right)>0$. Let $G_{L}^{\prime} \subset G_{L}$ denote those configurations for which the origin is part of $S\left(C_{0}\right)$. [Clearly $\operatorname{Prob}_{p}\left(G_{L}^{\prime}\right)>0$ since, with nonzero probability, every bond in $C_{0}$ is ferromagnetic.] 


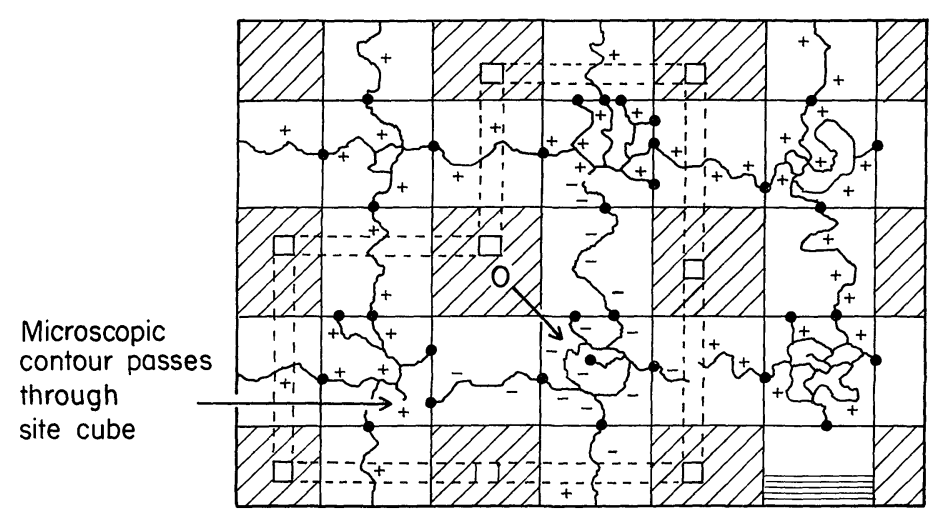

Fig. 6. Coarse-grained Peierls contour

Given $J \in G_{L}^{\prime}$, we delete from the (full $d$-dimensional) lattice all ferromagnetic bonds which do not participate in the event $G_{L}^{\prime}$. Let $R(J)$ denote the resulting configuration. By the GKS inequality [36], it suffices to estimate $\left\langle\sigma_{0}\right\rangle_{\beta,+}(R(J))$.

Thus consider $R(J), J \in G_{L}^{\prime}$, restricted to a finite volume $\Lambda \subset \mathbb{Z}^{d}$. In any spin configuration in which $\sigma_{0}=-1$, there is a collection $\left\{C_{i}\right\}$ of cubes, each of which contains a minus spin in the connected ferromagnetic component of the origin (see Fig. 6). Clearly $\left\{C_{i}\right\}$ is a connected set, and hence is surrounded by a contour $\Gamma$ on $\mathbb{V}^{*}$. This allows us to do a Peierls-Griffiths estimate.

For every three steps of the contour $\Gamma$ across bonds in which $\mathscr{B}_{i j}$ occurs, the corresponding microscopic contour must cross at least one ferromagnetic bond. (The worst case is when the microscopic contour passes through a "site" cube-see example in Fig. 6.) This provides the energy estimate

$$
\mathscr{E}_{\Gamma}(R(J)) \geqq \frac{1}{3} \beta J|\Gamma| .
$$

The contour entropy (i.e., the number of microscopic contours consistent with a macroscopic contour) is bounded by an estimate of the form

$$
\#\{\gamma \mid \gamma \in \Gamma\} \leqq e^{\alpha|\Gamma|}
$$

where $\alpha=\alpha(L, d)$. Finally, the number of coarse-grained contours of a given length is bounded above by the usual two-dimensional Peierls estimate. Let us define $\alpha^{\prime}=\alpha+c_{2}$.

Combining these estimates, we have

$$
\begin{aligned}
m(\beta) & \geqq \operatorname{Prob}_{p}\left(G_{L}^{\prime}\right) \lim _{\Lambda \nearrow \mathbb{Z}^{d}} \int\left\langle\sigma_{0}\right\rangle_{\beta, \Lambda^{+}}(R(J)) d \mu_{p}\left(J \mid G_{L}^{\prime}\right) \\
& \geqq \operatorname{Prob}_{p}\left(G_{L}^{\prime}\right)\left[1-2 \sum_{|\Gamma|=4}^{\infty} e^{-\left(\beta J / 3-\alpha^{\prime}\right)|\Gamma|}\right] .
\end{aligned}
$$

The lower bound is positive for $\beta$ large enough.

As in Sect. 4, this implies:

Corollary. For $p>\hat{p}_{c}^{\infty}$ and $\beta$ sufficiently large, the infinite cluster is a.s. magnetized, with constant magnetization. 
We note again that this result suffices to establish the a.s. existence of at least two Gibbs states.

\section{Two-Dimensional Systems with Antiferromagnetism}

\section{A. General Considerations}

It has already been shown that dilute ferromagnetic systems have at least two Gibbs states a.s. provided that the density of ferromagnetic bonds is above the percolation threshold and that $\beta$ is large enough. The question we address here is whether this behavior persists for dilute two-dimensional systems with some antiferromagnetic couplings. To be specific, let us consider a system with i.i.d. nearest neighbor couplings which can assume only three values:

$$
J_{i j}=\left\{\begin{array}{cl}
J_{F}, & \text { with probability } p, \\
0, & \text { with probability } 1-p-\varepsilon, \\
-J_{A}, & \text { with probability } \varepsilon .
\end{array}\right.
$$

It is expected that the behavior of the system will depend crucially on the relative density of the couplings. The decisive factor is again percolation, on the basis of which we may distinguish three ${ }^{5}$ cases:

(1) $p+\varepsilon<p_{c}$,

(2) $p<p_{c}, \varepsilon<p_{c}, p+\varepsilon>p_{c}$,

(3) $p>p_{c}, \varepsilon<p_{c}$.

It is easy to show that systems in the first class exhibit no long-range order and hence have a unique Gibbs state for all $\beta$; they are rather uninteresting, except perhaps for the presence of some Griffiths singularities [20].

We have no rigorous results on the phase structure of systems of the second type. However, these may provide realizations of somewhat frustrated random field Ising models [see Subsect. C(ii)].

The principal results of this section concern systems of the third type, with the additional assumption that the antiferromagnetic density and/or coupling strength is small. Under such circumstances, one expects persistence of "ferromagnetic behavior." Indeed, we shall show that there are a.s. at least two Gibbs states at low enough temperature. Moreover, we obtain bounds, in terms of the percolation correlation length, on the amount of antiferromagnetism that the system can tolerate without destroying the Curie transition.

These results are derived by showing that the ferromagnetic infinite cluster exhibits spontaneous magnetization. By the ferromagnetic infinite cluster, we mean that those sites of $\mathbb{Z}^{2}$ which are part of an infinite path of exclusively ferromagnetic bonds. An equivalent formulation is as follows: Consider the construction of the coupling configurations as a two-stage process. First, the ferromagnetic bonds are distributed at density $p$. Then, each bond which is not ferromagnetic is examined and, with probability $\varepsilon /(1-p)$, an antiferromagnetic

5 In $d=2$, the Kesten theorem [24] (i.e., $p_{c}=1 / 2$ ) excludes the fourth natural case, namely $p>p_{c}$ and $\varepsilon>p_{c}$ 
bond is placed there. The ferromagnetic infinite cluster is the set of bonds which were part of the infinite cluster before the second stage of the construction.

As a corollary to the magnetization of the infinite cluster, we show positivity of the Edwards-Anderson order parameter under the same circumstances. This does not, of course, imply positivity of the spontaneous magnetization - even at zero temperature. The latter is demonstrated only for $\varepsilon$ sufficiently small. Moreover, in this limit, we obtain the first order correction to the nominal spontaneous magnetization with + boundary conditions.

Although our principal analysis is restricted to systems with small amounts of antiferromagnetism, it suggests the possibility of rather bizarre phases when larger amounts of antiferromagnetism are present. In Subsect. C(i), we consider a model of a ferrite which has an antimagnetized phase [i.e., $\left.m^{+}(\beta)<0\right]$. The dynamical behavior of such a system is discussed. Whether antimagnetization occurs in the $\mathbb{Z}^{2}$ Bernoulli system remains an open problem.

\section{B. Systems with Antiferromagnetic Dust}

(i) Existence of Long-Range Order. We now extend the results of Sect. 4 to systems with a small amount of antiferromagnetism. The scheme is to employ the coarse-grained Peierls estimate used previously, with an additional requirement on good coupling configurations. Recall that a contour was said to be good if it crossed a ferromagnetic seal upon entering (and leaving) some fraction of the boxes through which it passed. When there is antiferromagnetism present, we must also be concerned with the behavior of the contour within the box; namely, we must control how much antiferromagnetism it typically encounters. This can be done by controlling either the relative coupling strength or the density.

The first alternative is technically easy, but physically unrealistic. Clearly, if a box contains $k$ antiferromagnetic bonds of strength $J_{A}$, then a contour can lose no more than $O\left(k \beta J_{A}\right)$ in energy while wandering through the box. Thus, if $L$ is large enough so that $\operatorname{Prob}\left(G_{L}\right)>1 / 2$, then the condition $\frac{J_{F}}{J_{A}} \frac{1}{L^{2}} \geqq O(1)$ ensures that the infinite cluster will magnetize, even if all nonferromagnetic bonds are antiferromagnetic. However, for $L \gg 1$ (i.e. $p$ near $p_{c}$ ), such a condition requires $J_{A} \ll J_{F}$, which is not experimentally realizable. Henceforth, we will assume without loss of generality that $\frac{J_{F}}{J_{A}} \frac{1}{L^{2}}<1$.

A physically more interesting question is, then, for fixed $J_{A} / J_{F}$, what density $\varepsilon$ of antiferromagnetic bonds can be tolerated by the system. We therefore fix, once and for all, the ratio $R=\frac{1}{8} \frac{J_{F}}{J_{A}}$. (The factor of 8 is for numerical convenience.) The relative amount of antiferromagnetism encountered within a box is measured by the parameter

$$
\lambda \equiv \frac{J_{A}}{J_{F}} \varepsilon L^{2}=\frac{8}{R} \varepsilon L^{2} .
$$

Our goal is to show that if $\lambda$ is not too large, the system exhibits ferromagnetic behavior. As will be shown, this indicates that the critical antiferromagnetic 
tolerance is bounded above by $\varepsilon_{0}=O\left(\xi^{-2}(p)\right)$ as $p \searrow p_{c}$. We start with the following elementary calculation.

Proposition 6.1. Let $n_{A}$ denote the number of antiferromagnetic bonds in an arrangement of $N$ boxes. Then

$$
\operatorname{Prob}\left(n_{A} \geqq R N\right) \leqq e^{-a(\lambda) N}
$$

with $a(\lambda) \rightarrow \infty$ as $\lambda \rightarrow 0$.

Proof. Recall that the boxes contain $4 L^{2}$ sites and hence no more than $8 L^{2}$ bonds. Furthermore, by assumption,

$$
1>\frac{J_{F}}{J_{A}} \frac{1}{L^{2}}=\frac{R}{8 L^{2}} \equiv x .
$$

Thus

$$
\operatorname{Prob}\left(n_{A} \geqq R N\right) \leqq \sum_{k \geqq R N}^{8 N L^{2}}\left(\begin{array}{c}
8 N L^{2} \\
k
\end{array}\right) \varepsilon^{k}(1-\varepsilon)^{8 N L^{2}-k} .
$$

For $\lambda<1, R N$ is larger than the mean number of antiferromagnetic bonds in box $(=\lambda R N)$, which implies that the sum is dominated by its first term. Performing the usual asymptotics, we obtain

$$
\begin{aligned}
\operatorname{Prob}\left(n_{A}>R N\right) & \leqq \frac{\left(8 L^{2} N\right)^{8 L^{2} N} \varepsilon^{R N}}{(R N)^{R N}\left(8 L^{2} N-R N\right)^{\left(8 L^{2} N-R N\right)}} \\
& =\left[\frac{\lambda}{(1-x)^{(1-x) / x}}\right]^{R N} \leqq(\lambda e)^{R N}
\end{aligned}
$$

Let us define an arrangement of $N$ boxes to be weakly antiferromagnetic if it contains fewer than $R N$ antiferromagnetic bonds. The set of configurations in which all arrangements are weakly antiferromagnetic will be denoted by $G_{\lambda}^{A}$. For $\lambda$ small, it is these configurations which dominate:

Proposition 6.2. As $\lambda \rightarrow 0, \operatorname{Prob}\left(G_{\lambda}^{A}\right) \rightarrow 1$.

Proof. This follows from Proposition 6.1 and (as in the proof of Proposition 4.2) from an exponential bound on the number of arrangements. We have

$$
1-\operatorname{Prob}\left(G_{\lambda}^{A}\right) \leqq \sum_{N=1}^{\infty} e^{a_{2} N}(\lambda e)^{R N} .
$$

Let us relate the previous proposition to a statement on the critical antiferromagnetic tolerance. We now have two notions of good configurations: (1) ferromagnetically good configurations, here denoted by $G_{L}^{F}$, and (2) weakly antiferromagnetic configurations, $G_{\lambda}^{A}$. By Proposition 4.2, whenever $p>p_{c}$, the former can be made arbitrarily probable by taking $L$ sufficiently large. Indeed, as noted earlier (cf. Remark following Theorem 4.3), we need only take $L$ on the scale of the correlation length, $\xi(p)$. By the previous proposition, weakly antiferromagnetic configurations are probable whenever $\lambda$ is small enough. Thus, if $L=O(\xi(p))$, this requires $\varepsilon<\varepsilon_{0}(p)$ with $\varepsilon_{0}(p)=($ const $) \xi^{-2}(p)$ for some small, but $p$-independent constant. Combining these ingredients, we have: 
Theorem 6.3. Suppose $p>p_{c}$ and $\varepsilon<\varepsilon_{0}(p)=O\left(\xi^{-2}(p)\right)$. Then, for $\beta$ sufficiently large, the ferromagnetic infinite cluster is a.s. magnetized.

Proof. For any $i \in \mathbb{Z}^{2}$, let $\mathscr{C}_{\infty}^{F}(i)$ denote the event that the site $i$ is in the infinite ferromagnetic cluster. The magnetization of the infinite ferromagnetic cluster in configuration $J$ is defined ${ }^{6}$ by

$$
\begin{aligned}
m_{\infty}^{F}(\beta, J) & \equiv \liminf _{\Lambda \ngtr \mathbb{Z}^{2}} \max _{\alpha} \sum_{i \in \Lambda} \chi_{\mathscr{C}_{\infty}^{F}(i)}(J)\left\langle\sigma_{i}\right\rangle_{\beta, \Lambda^{\alpha}}(J) / \sum_{i \in \Lambda} \chi_{\mathscr{C}_{i}^{F}(i)}(J) \\
& \geqq \liminf _{\Lambda \ngtr \mathbb{Z}^{2}} \sum_{i \in \Lambda} \chi_{\mathscr{C}_{\infty}(i)}(J)\left\langle\sigma_{i}\right\rangle_{\beta, \Lambda^{+}}(J) / \sum_{i \in \Lambda} \chi_{\mathscr{C}_{\infty}^{F}(i)}(J) \\
& \equiv m_{F, \infty}^{+}(\beta, J) .
\end{aligned}
$$

In the above, the maximum is taken over all boundary conditions on $\Lambda$, and $\Lambda \uparrow \mathbb{Z}^{2}$ through a van Hove sequence.

As in the proof of Theorem 2.3, we deal directly with the quantities $\left\langle\sigma_{i}\right\rangle_{\beta, \Lambda^{+}}$. To this end, let $\Lambda \subset 2 L \mathbb{Z}^{2}$ and define the set of arrangements $\Gamma(i)$ to be all finite, connected collections of boxes surrounding or containing the site $i \in \Lambda$. This, in turn, allows us to define ferromagnetically good and weakly antiferromagnetic configurations with respect to the site $i$, denoted by $G_{L}^{F}(i)$ and $G_{\lambda}^{A}(i)$, respectively.

Let $\bar{\Lambda}$ denote the union of $\Lambda \subset 2 L \mathbb{Z}^{2}$ with the surrounding annulus of width $2 L$. Now consider a configuration $J \in \mathscr{C}_{\infty}^{F}(i) \cap G_{L}^{F}(i) \cap G_{\lambda}^{A}(i)$, restricted to the box $\bar{\Lambda}$ with + boundary conditions. It is clear that every contour $\gamma \in \Gamma_{K}^{M}(i)$ has an energy estimate

$$
\mathscr{E}_{J}(\gamma) \geqq 2 \beta\left(M J_{F}-4 M R J_{A}\right)=\beta J_{F} M
$$

Thus the standard Peierls-Griffiths estimate shows that

$$
\begin{aligned}
\left\langle\sigma_{i}\right\rangle_{\beta, \bar{A}^{+}}(J) & \geqq 1-8 \sum_{M=1}^{\infty} e^{-\left(\beta J_{F}-\bar{c} L^{2}\right) M} \\
& \equiv s(\beta, L) \text { for } J \in \mathscr{C}_{\infty}^{F}(i) \cap G_{L}^{F}(i) \cap G_{\lambda}^{A}(i) .
\end{aligned}
$$
have

Denoting the complements of the events $G_{L}^{F}(i)$ and $G_{\lambda}^{A}(i)$ by $B_{L}^{F}(i)$ and $B_{\lambda}^{A}(i)$, we

$$
\begin{aligned}
m_{\infty}^{F}(\beta, J) & \geqq m_{F, \infty}^{+}(\beta, J) \\
& \geqq \lim _{\Lambda \nearrow \mathbb{Z}^{2}}\left[\sum_{i \in A} \chi_{\mathscr{C}_{\infty}^{F}(i)}\right]^{-1} \sum_{i \in A} \chi_{\mathscr{C}_{\infty}^{F}(i)}\left[s(\beta, L) \chi_{G_{L}^{F}(i)} \chi_{G_{\lambda}^{A}(i)}-\chi_{B_{L}^{F}(i)}-\chi_{B_{\lambda}^{A}(i)}\right] \\
& =s(\beta, L) \operatorname{Prob}\left(G_{L}^{F} \cap G_{\lambda}^{A} \mid \mathcal{O}_{\infty}^{F}\right)-\operatorname{Prob}\left(B_{L}^{F} \mid \mathcal{O}_{\infty}^{F}\right)-\operatorname{Prob}\left(B_{\lambda}^{A} \mid \mathcal{O}_{\infty}^{F}\right)
\end{aligned}
$$

a.s. by the Birkhoff ergodic theorem.

Finally, using the Harris-FKG inequality [38, 37], we find that a.s.

$$
m_{\infty}^{F}(\beta, J) \geqq s(\beta, L) \operatorname{Prob}\left(G_{L}^{F}\right) \operatorname{Prob}\left(G_{\lambda}^{A}\right)-\operatorname{Prob}\left(B_{L}^{F}\right)-\operatorname{Prob}\left(B_{\lambda}^{A}\right) .
$$

The lower bound is positive when $L$ is large enough [i.e., $L=O(\xi(p))$ ], if $\lambda$ is a small number [which is permitted when $\left.\varepsilon<\varepsilon_{0}(p)=O\left(\xi^{-2}(p)\right)\right]$, and finally, when $\beta$ is large enough.

6 Note that this is the analogue of the a.s. expression (2.11) for the total spontaneous magnetization 
Corollary. If $p>p_{c}$ and $\varepsilon<\varepsilon_{0}(p)=O\left(\xi^{-2}(p)\right)$, the Edwards-Anderson order parameter is a.s. positive for $\beta$ large enough.

Proof. In [2], the Edwards-Anderson order parameter $q_{\mathrm{EA}}$ was shown to be given by the a.s. expression

$$
q_{\mathrm{EA}}(\beta)=\lim _{\Lambda \nearrow \mathbb{Z}^{2}} \max _{\alpha}|\Lambda|^{-1} \sum_{i \in \Lambda}\left\langle\sigma_{i}\right\rangle_{\beta, \Lambda^{+}}^{2}(J)
$$

Theorem 6.3 shows that

$$
q_{\mathrm{EA}}(\beta) \geqq P_{\infty}(p) s^{2}(\beta, L) \operatorname{Prob}\left(G_{L}^{F}\right) \operatorname{Prob}\left(G_{\lambda}^{A}\right),
$$

which may be made positive.

(ii) The Spontaneous Magnetization. As stressed earlier, the existence of two or more Gibbs states does not imply positivity of the spontaneous magnetization. The most trivial example is that of a pure antiferromagnet below the Néel temperature for which $m(\beta) \equiv 0$. Nevertheless, it is reasonable to expect that for infinitesimal amounts of antiferromagnetism, the spontaneous magnetization should be positive, at least as the temperature tends to zero. This is established in Theorem 6.5.

By the above reasoning, it initially seems that the behavior of the spontaneous magnetization in a system with some antiferromagnetic couplings is of purely academic interest. Indeed, the Edwards-Anderson order parameter is certainly more appropriate for determining whether there is some type of magnetic longrange order. However, given that $q_{\mathrm{EA}}(\beta)>0$, the value of the spontaneous magnetization can be used to distinguished various types of magnetic order. In deterministic systems, there are three cases of relevance. First, if $m(\beta)$ assumes its nominal value, the order is purely ferromagnetic. At the other extreme is the case of pure antiferromagnetic order for which $m(\beta)=0$, as noted above. The generic case for mixed systems is that of ferrimagnetic order for which $m(\beta)$ is positive but smaller than its nominal value.

The spontaneous magnetization of a deterministic ferrite is calculated by examining the relative moments of the constituents of the Wigner-Seitz unit cell. In contrast, the spontaneous magnetization of random ferrites should be determined by effects on the scale of the percolation correlation length. This is the content of Theorem 6.6, in which we calculate the first order decrease in the nominal spontaneous magnetization of the plus state at zero temperature. If the plus state magnetization coincides with the true magnetization (which should be the case for $\varepsilon$ sufficiently small), this establishes the existence of ferrimagnetic order.

We begin with the following observation:

Lemma 6.4. For almost every configuration $J$,

$$
m(\beta)=m(\beta, J) \geqq P_{\infty}(p) m_{F, \infty}^{+}(\beta, J)-\left(P_{\infty}(p+\varepsilon)-P_{\infty}(p)\right),
$$

where $m_{F, \infty}^{+}(\beta, J)$ is the magnetization of the ferromagnetic infinite cluster in the plus state, as defined by Eq. (6.7).

Proof. Let $\Lambda \subset 2 L \mathbb{Z}^{2}$ and denote by $\mathscr{C}_{\Lambda}(i)$ the event that $i \in \Lambda$ is in the connected component of the boundary $\partial \Lambda$. Clearly $\left\langle\sigma_{i}\right\rangle_{\beta, \Lambda}=0$ if $J \notin \mathscr{C}_{\Lambda}(i)$. Similarly, let 
$\mathscr{C}_{\infty}^{F}(i)$ and $\mathscr{C}_{\infty}(i)$ denote the events that $i$ is in the ferromagnetic and total infinite clusters, respectively. Evidently,

$$
\begin{aligned}
m(\beta, J)= & \lim _{\Lambda \nearrow \mathbb{Z}^{2}} \max _{\alpha}|\Lambda|^{-1} \sum_{i \in \Lambda}\left\langle\sigma_{i}\right\rangle_{\beta, \Lambda^{\alpha}}(J) \chi_{\mathscr{C}_{\Lambda}(i)}(J) \\
\geqq & \liminf _{\Lambda \rtimes \mathbb{Z}^{2}}|\Lambda|^{-1} \sum_{i \in \Lambda}\left\langle\sigma_{i}\right\rangle_{\beta, \Lambda^{+}}(J) \chi_{\mathscr{C}_{\Lambda}(i)}(J) \\
= & \liminf _{\Lambda \rtimes \mathbb{Z}^{2}}|\Lambda|^{-1} \sum_{i \in \Lambda}\left\langle\sigma_{i}\right\rangle_{\beta, \Lambda^{+}}(J) \\
& \cdot\left[\left(\chi_{\mathscr{C}_{\Lambda}(i)}(J)-\chi_{\mathscr{C}_{\infty}(i)}(J)\right)+\chi_{\mathscr{C}_{\infty}(i)}(J)+\left(\chi_{\mathscr{C}_{\infty}(i)}(J)-\chi_{\mathscr{C}_{\infty}(i)}(J)\right)\right] .
\end{aligned}
$$

Let us examine the three terms separately. The first represents those sites which are in the connected component of the boundary, but are not connected to infinity. This is just a surface effect and vanishes in the limit $\Lambda>\mathbb{Z}^{2}{ }^{7}$ The second term, if multiplied and divided by $\sum_{i \in A} \chi_{\mathscr{C}_{\infty}(i)}(J)$, converges a.s. to $P_{\infty}(p) m_{F, \infty}^{+}(\beta, J)$, where $m_{F, \infty}^{+}(\beta, J)$ is the plus state magnetization of the ferromagnetic infinite cluster, the subject of Theorem 6.3. Ignoring the coefficient $\left\langle\sigma_{i}\right\rangle_{\beta, \Lambda^{+}}$(which we bound below by -1$)$, the third sum obviously converges a.s. to $P_{\infty}(p+\varepsilon)-P_{\infty}(p)$. For future reference, note that the individual terms $\left[\chi_{\mathscr{C}_{\infty}(i)}(J)-\chi_{\mathscr{C}_{\infty} F_{(i)}}(J)\right]$ have the interesting property that they are nonzero only if the site $i$ is connected to infinity exclusively through paths containing antiferromagnetic bonds.

We are now prepared to bound the (zero-temperature) magnetization by quantities familiar in percolation theory. In order that the final expression has a manageable form, we stipulate $J_{A}<J_{F}$ (cf. Remark following proof of Theorem $6.5)$.

Theorem 6.5. Suppose $p>p_{c}$ and $J_{A}<J_{F}$. Then

$$
\lim _{\beta \rightarrow \infty} m(\beta) \geqq P_{\infty}(p)-\varepsilon \frac{d P_{\infty}}{d p}+o(\varepsilon) .
$$

Note that this immediately implies:

Corollary. If $p>p_{c}$ and $J_{A}<J_{F}$, the zero-temperature spontaneous magnetization is positive for $\varepsilon$ sufficiently small.

Proof of Theorem 6.5. In $d=2$, for $p \neq p_{c}, P_{\infty}(p)$ is known to have derivatives of all orders [29]. Thus we may replace $P_{\infty}(p+\varepsilon)-P_{\infty}(p)$ by $\varepsilon P_{\infty}^{\prime}(p)+O\left(\varepsilon^{2}\right)$ in the lower bound of the previous lemma. It suffices to show that $\lim _{\beta \rightarrow \infty} m_{F, \infty}^{+}(\beta, J)=1+o(\varepsilon)$.

Recall that the difference between $m_{\infty}^{F}$ and 1 - in the limit $\beta \rightarrow \infty$-involves the probabilities of bad ferromagnetic and antiferromagnetic configurations [cf. Eq. (6.11)]. The former is independent of $\varepsilon$ and depends only, albeit exponentially, on the ratio $L / \xi(p)$ of the coarse-graining scale to the correlation length. The latter

7 In $d=2$, the probability that a given site is in a finite cluster of $n$ bonds is known to be bounded above [31] and below [41] by estimates of the form $e^{-c n^{1 / 2}}$ for all $p>p_{c}$. It is worth noting that for $d>2$, the expected behavior for $p>p_{c}$ is $e^{-c n^{(d-1) / d}}$. However, while a lower bound of this form has been established [41], an upper bound - which is what we would need here - has only been proved for $p$ near 1 [42]. For all $d$, the probability that a given site is in a cluster exceeding $n$ bonds is known [32] to decay exponentially (i.e., $e^{-c n}$ ) whenever $p<\pi_{c}$, with $\pi_{c}$ the point at which the expected cluster size diverges 
depends on $\varepsilon$ via $\lambda \sim \varepsilon L^{2}$. Clearly, we must rescale $L$ to drive the first quantity, $\operatorname{Prob}\left(B_{L}^{F}\right)$, to zero. Due to the exponential dependence on $L$, it suffices to take $L(\varepsilon) \sim \varepsilon^{-\delta}$ for some $\delta>0$. Unfortunately, this sends $\operatorname{Prob}\left(B_{\lambda(\varepsilon)}^{A}\right) \sim \lambda^{R}(\varepsilon) \sim\left(\varepsilon L^{2}(\varepsilon)\right)^{R}$ to zero sublinearly in $\varepsilon$, destroying the desired bound on the magnetization. Evidently, a little more care is required.

To this end, we modify our definition of weakly antiferromagnetic configurations. This is done by distinguishing two classes of contours: those that only visit boxes in the immediate vicinity of the origin, and those which are forced to break many ferromagnetic seals. The latter are handled by the usual criterion. In particular, we say that $J \in G_{\lambda}^{A, 1}$ if all arrangements of $N \geqq N_{1}>9$ boxes contain fewer than $R N$ antiferromagnetic bonds. We choose $N_{1}$ such that

$$
1-\operatorname{Prob}\left(G_{\lambda}^{A, 1}\right) \leqq \sum_{N \geqq N_{1}} e^{a_{2} N}(\lambda e)^{R N}=o(\varepsilon) .
$$

(The existence of such an $N_{1}$ is guaranteed provided that we take $\delta<1 / 2$.) Note that $N_{1}$ depends on $\delta$ and $R$, but is independent of $\varepsilon$.

The contours in the vicinity of the origin have always been handled by conditioning (as we are doing here) on the event that the origin is part of the infinite ferromagnetic cluster. However, in this case, we have the additional requirement $J_{A}<J_{F}$. To exploit this, let us choose $N_{2}$ to be the smallest integer such that all arrangements of fewer than $N_{1}$ boxes are contained in a block of $N_{2}$ boxes surrounding the origin. Then our energy estimate (6.8) for $N \leqq N_{2}$ remains legitimate in those configurations $J \in G_{\lambda}^{A}$, 2 with no more than one antiferromagnetic bond in the block of $N_{2}$ boxes. We have

$$
\operatorname{Prob}\left(G_{\lambda}^{A, 2}\right) \geqq 1-(\text { const })\left(\varepsilon N_{2} L^{2}\right)^{2}=1-o(\varepsilon),
$$

provided that $\varepsilon^{2} L^{4}(\varepsilon)=o(\varepsilon)$. Again, this may be guaranteed by taking $\delta$ small enough (e.g., $\delta=1 / 5$ ).

Thus, if we redefine weakly antiferromagnetic configurations by $\widetilde{G}_{\lambda}^{A}=G_{\lambda}^{A, 1} \cap G_{\lambda}^{A, 2}$, it is possible to drive both $\operatorname{Prob}\left(B_{L}^{F}\right)$ and $\operatorname{Prob}\left(\widetilde{B}_{\lambda}^{A}\right)$ to zero like $o(\varepsilon)$, while still retaining the original Peierls-Griffiths estimate.

Remark. The condition $J_{A}<J_{F}$ is necessary for the above theorem. In the opposite case, $J_{A} \geqq J_{F}$, there is another effect of order $\varepsilon$. This occurs when there is an antiferromagnetic bond lodged in the infinite cluster in the vicinity of the origin (see Fig. 7a). Under such circumstances, a sufficient condition for $\left\langle\sigma_{0}\right\rangle(J)>0$ is that the origin be multiply connected.

flumur antiferromagnetic bond ferromagnetic bond
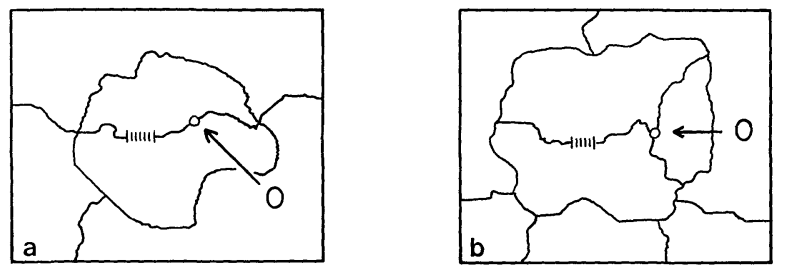

Fig. 7. a Antiferromagnetism lodged in infinite cluster. b Origin multiply connected 
In other words, in addition to the antiferromagnetic connection, there must be a number (depending on $J_{A} / J_{F}$ ) of disjoint purely ferromagnetic paths emanating from the origin (see Fig. 7b). It is possible (but nontrivial) to compute explicit bounds on $m(\beta)$ in, say, the case $J_{F} \leqq J_{A}<2 J_{F}$. However, it is clear that (1) the modifications in our previous expression are of order $\varepsilon$, and (2) these modifications are not related to any well-known quantities in percolation.

Next, we address the problem of showing that the antiferromagnetic dust actually lowers the zero-temperature nominal magnetization, and determining by how much. This requires an upper bound to complement the lower bound of Theorem 6.5. Since as $\varepsilon \rightarrow 0$, we expect that the magnetization is achieved by applying + boundary conditions ${ }^{8}$, we address the question only for the plus state.

In order to calculate the zero-temperature decrease, it is instructive to consider which percolation configurations contribute to the decrease. As noted earlier, the term $P_{\infty}(p+\varepsilon)-P_{\infty}(p)$ represents the contribution of those sites which rely crucially on antiferromagnetic bonds to achieve a connection to infinity. Let us see how this situation can arise in terms of the two-stage process outlined in Subsect. A. To first order in $\varepsilon$, this occurs at the site $i$ only if (1) in the purely ferromagnetic configuration, the site $i$ is in a finite cluster which would be connected to the infinite cluster if we were to insert a single bond in a crucial location, and (2) an antiferromagnetic bond is inserted in that location. The "crucial bond" referred to above is precisely an articulation (or pivotal) bond, a notion introduced by Russo $[43,44]$ to describe a bond upon whose occupation a particular event depends. If the situation described in (1) occurs at the bond $b$, we say that $b$ is an articulation bond for the event $\mathscr{C}_{\infty}^{F}(i)$ in configuration $J$. The set of configurations in which $b$ is an articulation bond for $\mathscr{C}_{\infty}^{F}(i)$ will be denoted by $\delta\left[\mathscr{C}_{\infty}^{F}(i)\right]_{b}$. The full event described above is therefore

$$
\delta\left[\mathscr{C}_{\infty}^{F}(i)\right]_{b}^{*} \equiv \delta\left[\mathscr{C}_{\infty}^{F}(i)\right]_{b} \cap\{b \text { is occupied by an antiferromagnetic bond }\} .
$$

Clearly, the two events comprising $\delta\left[\mathscr{C}_{\infty}^{F}(i)\right]_{b}^{*}$ are independent, so that $\operatorname{Prob}\left(\delta\left[\mathscr{C}_{\infty}^{F}(i)\right]_{b}^{*}\right)=\varepsilon \operatorname{Prob}\left(\delta\left[\mathscr{C}_{\infty}^{F}(i)\right]_{b}\right)$.

Let us calculate the probability that the site $i$ is connected to infinity exclusively through paths containing antiferromagnetic bonds. First, there is the probability that each of the paths contains only a single antiferromagnetic articulation bond. Alternating bounds on this are given by inclusion-exclusion:

$$
\begin{aligned}
\operatorname{Prob}\left(\bigcup_{b} \delta\left[\mathscr{C}_{\infty}^{F}(i)\right]_{b}^{*}\right)= & \varepsilon \sum_{b} \operatorname{Prob}\left(\delta\left[\mathscr{C}_{\infty}^{F}(i)\right]_{b}\right) \\
& -\sum_{b \neq b^{\prime}} \operatorname{Prob}\left(\delta\left[\mathscr{C}_{\infty}^{F}(i)\right]_{b}^{*} \cap \delta\left[\mathscr{C}_{\infty}^{F}(i)\right]_{b^{\prime}}^{*}\right)+\ldots
\end{aligned}
$$

By the ergodic theorem and the Russo formula [43], the first term above is exactly $\varepsilon \frac{d}{d p} \operatorname{Prob}\left(\mathcal{O}_{\infty}^{\boldsymbol{F}}\right) \equiv \varepsilon \frac{d P_{\infty}}{d p}$. The exclusion terms are of order $\varepsilon^{2}$ (and, indeed, finite due to the bounds on the finite cluster distribution [31] described earlier). To calculate

8 A proof of this natural conjecture seems to require uniform expansions in the presence of an external field, which should be possible under the conditions of the present work (i.e., $p>p_{c}, \varepsilon$ sufficiently small and $\beta$ sufficiently large) 
the complete effect, we must also compute the probability that each of the paths contains two or more antiferromagnetic bonds (i.e., double and higher order articulation events). This is clearly of order $\varepsilon^{2}$. Moreover, when combined with the various "exclusion pieces" previously discarded, this generates term by term the higher order derivatives of $P_{\infty}(p)$. Indeed, a detailed knowledge of the expansion of $P_{\infty}(p)$ seems to be necessary for the analysis of nonperturbative effects of antiferromagnetic impurities (see Subsect. C).

Given the foregoing discussion, we can now establish our final result:

Theorem 6.6. Suppose $p>p_{c}$ and $J_{A}<J_{F}$. Then for a.e. configuration $J$,

$$
P_{\infty}(p)-\varepsilon \frac{d P_{\infty}}{d p}+o(\varepsilon) \geqq \lim _{\beta \rightarrow \infty} \bar{m}^{+}(\beta, J) \geqq \lim _{\beta \rightarrow \infty} \underline{m}^{+}(\beta, J) \geqq P_{\infty}(p)-\varepsilon \frac{d P_{\infty}}{d p}+o(\varepsilon),
$$

where $\bar{m}^{+}(\beta, J)$ and $\underline{m}^{+}(\beta, J)$ are the upper lower limits of the plus state magnetization, i.e.,

$$
\begin{aligned}
& \bar{m}^{+}(\beta, J) \equiv \limsup _{\Lambda \mp \mathbb{Z}^{2}}|\Lambda|^{-1} \sum_{i \in \Lambda}\left\langle\sigma_{i}\right\rangle_{\beta, \Lambda^{+}}(J), \\
& \underline{m}^{+}(\beta, J) \equiv \liminf _{\Lambda \mp \mathbb{Z}^{2}}|\Lambda|^{-1} \sum_{i \in \Lambda}\left\langle\sigma_{i}\right\rangle_{\beta, \Lambda^{+}}(J) .
\end{aligned}
$$

Proof. The lower bound is an immediate consequence of Theorem 6.5.

In order to prove the upper bound, we focus on those sites which are connected exclusively through a single antiferromagnetic articulation bond. These sites will provide the first order correction to the nominal zero-temperature magnetization $P_{\infty}(p)$. To demonstrate that the correction is indeed $-\varepsilon P_{\infty}^{\prime}(p)+o(\varepsilon)$, we shall gauge transform up to the articulation bond, and determine the magnetization of the transformed system using the "good-bad" estimates of Theorem 6.5.

By the previous discussion, the probability that a given site $i$ is connected to infinity exclusively through a single antiferromagnetic articulation bond is $\varepsilon P_{\infty}^{\prime}(p)+O\left(\varepsilon^{2}\right)$ [cf. Eq. (6.17)]. If we further require that the articulation bond occurs within the first nine boxes surrounding the site $i$, this probability is modified by an additive factor of $e^{-O(\sqrt{L})}$, according to the finite cluster size distribution bounds $[31,41]$. Let us denote by $A_{L}(i)$ the above described event. We have

provided that $L(\varepsilon) \sim \varepsilon^{-\delta}, \delta>0$.

$$
\operatorname{Prob}\left(A_{L}(i)\right)=\varepsilon \frac{d P_{\infty}}{d p}+O\left(\varepsilon^{2}\right)
$$

In order to do the "good-bad" estimates of Theorem 6.5, let us restrict attention to good configurations $J \in G_{L}^{F}(i) \cap \widetilde{G}_{\lambda}^{A}(i)$. Denoting the event of interest by

$$
\mathscr{A}_{L, \lambda}(i)=A_{L}(i) \cap G_{L}^{F}(i) \cap \widetilde{G}_{\lambda}^{A}(i),
$$

subadditivity gives us

$$
\operatorname{Prob}\left(\mathscr{A}_{L, \lambda}(i)\right) \geqq \operatorname{Prob}\left(A_{L}(i)\right)-\operatorname{Prob}\left[\left(G_{L}^{F}(i) \cap \widetilde{G}_{\lambda}^{A}(i)\right)^{c}\right] .
$$

By Theorem 6.5 and Eq. (6.18), this implies

$$
\operatorname{Prob}\left(\mathscr{A}_{L, \lambda}(i)\right) \geqq \varepsilon \frac{d P_{\infty}}{d p}+o(\varepsilon),
$$

provided that we choose $\delta$ properly (e.g., $\delta=1 / 5$ ). 
Consider a configuration $J \in \mathscr{A}_{L, \lambda}(i)$, restricted to a large regular box $\Lambda \subset 2 L \mathbb{Z}^{2}$. We claim that

$$
\left\langle-\sigma_{i}\right\rangle_{\beta, \Lambda^{+}} \geqq 1-e^{-O(\beta)},
$$

uniformly in $|\Lambda|$, for $\beta$ sufficiently large. To see this, gauge transform all spins in the connected cluster of $\sigma_{i}$ up to the articulation bond. Then (6.22) is precisely the Peierls-Griffiths estimate of Theorem 6.5, performed on the gauge transformed system.

To prove the desired inequality, we now write $\bar{m}^{+}$as the sum of several terms, reminiscent of Eq. (6.13):

$$
\begin{aligned}
\bar{m}^{+}(\beta, J)= & \lim _{\Lambda} \sup _{\mathbb{Z}^{2}}|\Lambda|^{-1} \sum_{i \in \Lambda}\left\langle\sigma_{i}\right\rangle_{\beta, \Lambda^{+}}(J)\left[\left(\chi_{\mathscr{C}_{\Lambda}(i)}(J)-\chi_{\mathscr{C}_{\infty}(i)}(J)\right)\right. \\
& \left.+\chi_{\mathscr{C}_{\infty} F_{\infty}(i)}(J)+\chi_{\mathscr{A}_{L, \lambda}(i)}(J)+\left(\chi_{\mathscr{C}_{\infty}(i)}(J)-\chi_{\mathscr{C}_{\infty}(i)}(J)-\chi_{\mathscr{A}_{L, \lambda}(i)}(J)\right)\right] .
\end{aligned}
$$

As before, the first term vanishes as $\Lambda \rtimes \mathbb{Z}^{2}$, and (bounding $\left\langle\sigma_{i}\right\rangle \leqq 1$ ) the second converges a.s. to $P_{\infty}(p)$. By the discussion preceding the theorem, the fourth term is of order $O\left(\varepsilon^{2}\right)$. Our principal concern is the third term. For this, we bound $\left\langle\sigma_{i}\right\rangle_{\beta, A^{+}}$ above according to the estimate (6.22). By ergodicity, the remaining sum converges a.s. to $\operatorname{Prob}\left(\mathscr{A}_{L, \lambda}(i)\right)$, which we bound by Eq. (6.21).

Remark. As noted earlier, the principal distinction between $d=2$ and $d>2$ is that in the former case $1-\pi_{c}^{*}=p_{c}$, i.e. whenever the infinite cluster is present, the dual clusters are of finite expected size. It should be clear that the analysis presented here extends to $d>2$ whenever $p>1-\pi_{c}^{*}$.

\section{Nonperturbative Effects of Antiferromagnetism: Open Problems and Speculations}

(i) The Random Antiferrimagnetic Phase. The previous subsection characterizes the behavior of two-dimensional systems above the ferromagnetic threshold in the presence of small amounts of antiferromagnetism. It is amusing to speculate about the effects of large amounts of antiferromagnetism. It is probable that once $\varepsilon \xi^{2}(p) J_{A} / J_{F}$ is of order unity, frustration becomes important. Eventually, effects of this sort may disrupt the ferromagnetic order of the infinite cluster. (Whether or not ordering of some type persists is not clear.) Systems with substantial frustration - that is, spin glasses - are purported to exhibit dramatic dynamic behavior.

Here we suggest a different mechanism by which ferrimagnetic systems can exhibit path-dependent dynamics. In order to distinguish this effect from that of frustration, it is convenient to assume that $J_{A} \ll J_{F} \xi^{-2}(p)$. This allows us to neglect the influence of antiferromagnetic bonds lodged within the infinite cluster. We emphasize, however, that while such an assumption simplifies the analysis, the effects discussed may occur even if the assumption is violated.

We have already seen (Theorem 6.6) that the first order effect of antiferromagnetism is to decrease the plus state magnetization due to antiferromagnetic connections of finite clusters to the (ferromagnetic) infinite cluster. This raises the intriguing possibility that under certain conditions there is bulk antimagnetization, i.e. the infinite cluster is magnetized, but the overall magnetization is in the 


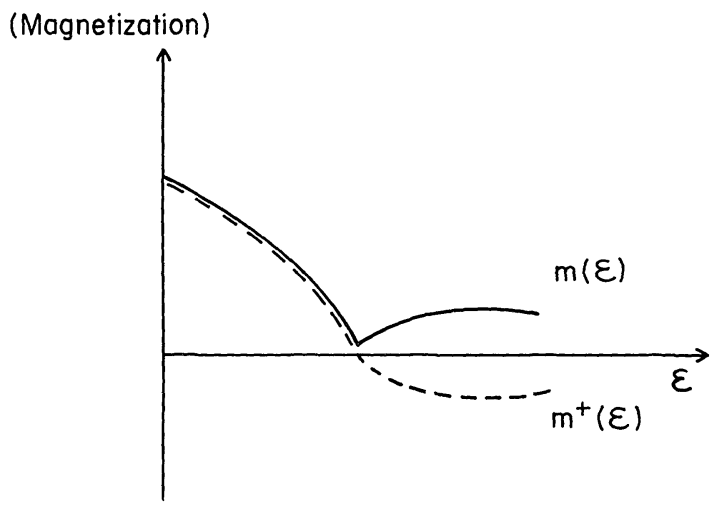

Fig. 8. Hypothetical behavior of random ferrites

opposite sense. That such a situation is possible follows from the observation that for $p \gtrsim p_{c}$, the density of ferromagnetic bonds in the infinite cluster is $P_{\infty}(p) \ll 1,{ }^{9}$ while the density of those in finite clusters is $p-P_{\infty}(p) \simeq p=O(1)$. Thus, if the predominant effect of antiferromagnetism is to connect finite clusters to the infinite cluster through an odd number of antiferromagnetic bonds, it is possible to have more spins aligned against the infinite cluster than aligned with it. Whether the proviso of the previous sentence is satisfied (i.e., whether more spins are connected through an odd number of antiferromagnetic bonds) represents a challenging nonperturbative question in percolation theory. Indeed, it requires an analysis of articulation events of all orders, as discussed in Subsect. B.

The existence of antimagnetized states is entirely consistent with thermodynamic considerations. Indeed, later in this section, we shall present a model of a random system which exhibits antimagnetization. First, however, let us examine, on a heuristic level, the thermodynamic and dynamic behavior of antimagnetized systems.

For simplicity, we restrict attention to only two Gibbs states: those generated by plus and minus boundary conditions. The distinguished thermodynamic property of an antimagnetized system is that the state obtained in the limit of infinitesimal positive external fields (i.e., $h \rightarrow 0^{+}$) disagrees with that generated by plus boundary conditions. This means that the spontaneous magnetization is not computed from plus boundary conditions, despite the presence of long-range ferromagnetic order. This is illustrated in Fig. 8, in which we give a schematic graph of the actual and plus state magnetizations, $m$ and $m^{+}$, of an idealized system as functions of the antiferromagnetic density $\varepsilon$. In this hypothetical system, there is a random ferrimagnetic phase in which $m$ and $m^{+}$agree, a random antiferrimagnetic phase of antimagnetized states and, finally, a completely frustrated phase with no ferromagnetic long-range order.

A purely thermodynamic consequence of antimagnetization should be the existence of a realignment transition at a critical value of the external field. To see this, let us start with (say) the state obtained from minus boundary conditions. This

9 Recall that $P_{\infty}(p)$ is known to be continuous in $d=2[43]$, so that $P_{\infty}(p) \ll 1$ for $p$ near $p_{c}$ 
state should be "stable" to a small, positive magnetic field since the disagreement between the infinite ferromagnetic cluster and the external field is more than compensated by the agreement between the field and the finite anticonnected clusters. However, for sufficiently large external field, the infinite cluster must align with the field. This implies that there is a critical field, $h_{r}$, which causes realignment. Moreover, since an infinite connected object must realign, we expect that the system has large scale inhomogeneities whenever $h$ is near $h_{r}$.

The above considerations suggest that antimagnetized systems exhibit interesting dynamics. Indeed, consider the dynamics as a strong, positive external field is taken to zero. If the field is removed adiabatically, the system presumably settles down into the state in which the majority of spins are plus, and hence the infinite cluster is minus. On the other hand, if the field is shut off instantaneously, one is compelled to argue that the system settles into the state in which the infinite cluster is plus magnetized. Indeed, while there is an infinite energy barrier against flipping the ferromagnetic cluster, the anticonnected finite clusters may easily flip, one at a time. The above, simple-minded example suggests that mechanisms other than frustration may be responsible for some of the observed path-dependent behavior of random magnetic systems.

It should be emphasized that as strange as the effects described above may seem, these effects are a natural (albeit nonrigorous) consequence of the existence of an antimagnetized state, which in turn depends exclusively on the nature of typical coupling configurations of the measure $d R(J)$. In other words, the question of whether there is a random antiferrimagnetic phase in the systems discussed here - and hence, of whether these effects can occur - is an issue in percolation theory, not statistical mechanics. For the $\mathbb{Z}^{2}$ Bernoulli system, the question is complicated by the fact that if the antiferrimagnetic phase occurs, it is likely to be an intermediate phase, and thus is difficult to study by perturbative techniques.

Below we consider percolation on a lattice consisting of two types of sites, with a structure typical of that which occurs in real ferrites. For this system, antiferrimagnetism occurs in an extreme (i.e., not intermediate) phase. We are thus able to use the perturbative techniques developed earlier to prove that the phase persists in the presence of random impurities.

The crystal, consisting of sites of type $a$ and $b$, is shown in Fig. 9. Each of the three types of nearest neighbor couplings $(a a, b b$, and $a b)$ is taken to be an i.i.d. random variable which can assume only three values, as in Eq. (6.1). Here, however, the values and densities are different for the different types of bonds, and
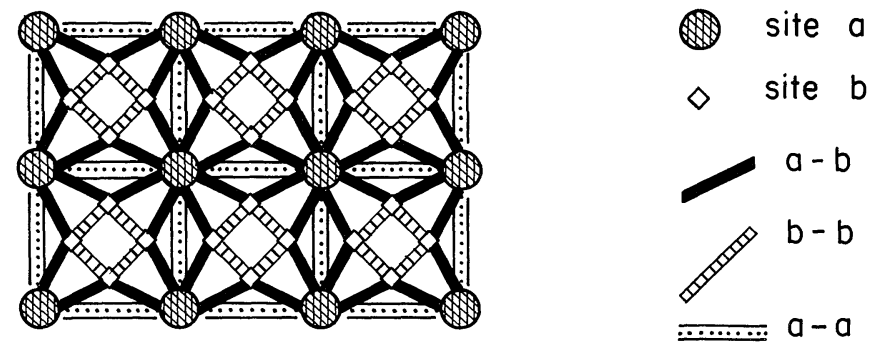

Fig. 9. A ferrite crystal 
will be distinguished by superscripts. When $p^{a a}=p^{b b}=p^{a b}=1$, the system is completely ferromagnetic with zero-temperature magnetization/cell $=+5$. When $p^{a a}=p^{b b}=\varepsilon^{a b}=1$, the system is antiferrimagnetic with zero-temperature magnetization/cell $=+3$. (Note that the plus state zero-temperature magnetization/cell $=-3$.) It follows that there is a realignment transition as we vary the $a b$ parameter. Moreover, the obvious (but tedious) extension of the techniques of Subsect. B shows that the behavior of the system in either of the above two limits is stable to random impurities.

(ii) Relationship to the Random Field Ising Model. It has been argued [27, 45-48] that random magnetic systems provide experimental realizations of the random field Ising model (RFIM), in the sense that a random system in a uniform field may be mapped into a purely ferromagnetic system in a random field. For example [46], if the random magnetic system has i.i.d. site disorder:

$$
\mathscr{H}=-J \sum_{\langle i, j\rangle} \tau_{i} \tau_{j} \sigma_{i} \sigma_{j}-h \sum_{i} \sigma_{i},
$$

the mapping is accomplished via a straightforward gauge transformation:

$$
\overline{\mathscr{H}}=-J \sum_{\langle i, j\rangle}\left|\tau_{i}\right|\left|\tau_{j}\right| s_{i} s_{j}-h \sum_{i} \frac{\tau_{i}}{\left|\tau_{i}\right|} s_{i} .
$$

In the special case in which the $\tau_{i}$ have mean exactly zero, and a perfectly symmetric distribution about the mean, the transformed system is indeed an RFIM. However, this result is of questionable physical interest since it seems to depend on the above two conditions, neither of which are experimentally realizable.

A more interesting set of examples was proposed by Fishman and Aharony [27], who argued that any random system which exhibits antiferromagnetic ordering can be mapped into an RFIM. However, the transformation is not exact; it induces short range correlations between the "random" fields and effective couplings which are argued to be irrelevant near the critical point.

Given that the mapping of Fishman and Aharony is not exact, and that the correlations may tend to order the transformed systems relative to the RFIM, it is of some interest to study the untransformed systems. Indeed, among the untransformed systems considered in [27] are those for which the coupling distribution satisfies $J_{i j}<-J_{A}<0$. These will clearly order at zero temperature in a small uniform field in $d=2$, whereas such ordering is not expected in $d=2$ for the RFIM [45]. Having dispensed with this rather obvious set of (zero-temperature) counterexamples, the interesting question is to determine which other coupling distributions satisfy the Fishman and Aharony criterion (i.e., which distributions produce antiferromagnetic order in the absence of an external field). This can be ascertained by transforming the results of the previous sections. In particular, assuming $p_{c}=\hat{p}_{c}^{\infty}$, the necessary and sufficient condition for systems with only dilution impurities is that the antiferromagnetic density exceed the percolation threshold. For two-dimensional systems with some ferromagnetic impurities, it is sufficient that the antiferromagnetic bonds percolate and that the ferromagnetic density be less than $\varepsilon_{0}(p)=O\left(\xi^{-2}(p)\right)$. 
We have no results on whether a field alters the lower critical dimension of the systems discussed above. However, in order to illustrate the purported relationship of the systems considered in this paper (and their gauge transforms) to the RFIM, below we provide a heuristic mapping of an antiferromagnetic system with dilution impurities. We caution the reader that this mapping does not produce a "true" RFIM.

Consider a system with i.i.d. nearest neighbor coupling distribution

$$
J_{i j}=\left\{\begin{array}{cl}
-J_{1} & \text { with probability } p \\
-J_{2} & \text { with probability } \varepsilon \\
0 & \text { with probability } 1-p-\varepsilon,
\end{array}\right.
$$

with $p \lesssim p_{c}$ and $\varepsilon \ll 1$, but $p+\varepsilon>\hat{p}_{c}^{\infty}$. Assume further that $\left|J_{1}\right| \gg\left|J_{2}\right|$. The structure of the configurations is most easily visualized in terms of the two-step process outlined earlier. First, bonds of strength $-J_{1}$ are distributed with density $p$. Since $p \lesssim p_{c}$, these bonds form large, but finite clusters. Next, antiferromagnetism of strength $-J_{2}$ is distributed with density $\varepsilon /(1-p)$ among the unoccupied bonds. The effect is to connect an infinite subcollection of the $-J_{1}$ clusters, $\mathscr{C}_{1}, \ldots, \mathscr{C}_{k}, \ldots$, to form an infinite cluster. By Theorem 4.3, in the absence of a field the system exhibits antiferromagnetic ordering with (at least) two ordered states. For definiteness, let us consider the state in which the sublattice containing the origin is up. Denoting this sublattice by $\mathbb{L}_{1}$ and the other by $\mathbb{L}_{2}$, we may define lattice indicators

$$
l_{i}= \begin{cases}+1, & i \in \mathbb{L}_{1}, \\ -1, & i \in \mathbb{L}_{2},\end{cases}
$$

and the cluster spin variables

$$
S_{k}=\frac{1}{\left|\mathscr{C}_{k}\right|} \sum_{i \in \mathscr{C}_{k}} l_{i} \sigma_{i}
$$

If $\left|J_{1} \beta\right| \gg 1$ while $\left|\beta J_{2}\right|=O(1)$, then with overwhelming probability, the clusters will be frozen and the spin degree of freedom will be accurately described by the $\left\{S_{k}\right\}$. For this range of parameters, we may integrate out all other degrees of freedom to obtain an effective Hamiltonian in the $\left\{S_{k}\right\}$ variables with ferromagnetic interactions. (Furthermore, for $\varepsilon$ sufficiently small, the vast majority of the interactions are pairwise.) The effect of a uniform magnetic field on the original variables is two-fold. First, it alters the nature of the effective interaction between cluster variables in an essentially uncontrolled fashion - indeed, it is this effect which could invalidate the mapping into an RFIM. The second effect, however, is quite clear. The cluster variables $\left\{S_{k}\right\}$ couple linearly to a random field given by

$$
H_{k}=h \sum_{i \in \mathscr{C}_{k}} l_{i}
$$

where $h$ is the strength of the uniform field applied to the original system. Note that the $H_{k}$ are i.i.d. random variables with mean zero (and, of equal importance, with distribution symmetric about zero). Thus, the fluctuating degrees of freedom are 
described by an effective Hamiltonian of the form

$$
\begin{aligned}
\mathscr{H}_{\text {eff }} & =\mathscr{H}_{0}+\sum_{k} H_{k} S_{k}, \\
\mathscr{H}_{0} & =-\sum_{\langle i, j\rangle} J_{i j}^{\text {eff }} S_{i} S_{j}+\text { higher body terms } .
\end{aligned}
$$

Again, we emphasize that the $J_{i j}^{\text {eff }}$ are not simply the ferromagnetic couplings one obtains in the absence of an external field.

The previous example suggests another class of random magnetic systems, not considered by Fishman and Aharony, which are candidates for realizations of the RFIM. Consider systems similar to those just analyzed in which the predominant antiferromagnetic coupling has been replaced by a ferromagnetic coupling $J_{F}$. (These are just the case 2 systems of Subsect. A.) Here, the ferromagnetic bonds form large clusters of finite expected size. The addition of antiferromagnetic bonds has two effects. First, these bonds may lodge within the ferromagnetic clusters, an effect which should be controllable provided that $\left|J_{A} / J_{F}\right|$ is sufficiently small on the scale of $p-p_{c}$. Second, the antiferromagnetic bonds will connect an infinite collection of these ferromagnetic clusters to form the $(p+\varepsilon)$-infinite cluster.

For $\beta$ such that $\beta J_{F} \gg 1$ while $\beta J_{A}=O(1)$, the ferromagnetic clusters may be accurately described by "block" variables. This raises the interesting possibility that the block variables exhibit long-range order for some regime of parameters. Were this to occur, we would not classify it as spin glass order. Indeed, such order should only occur if it is possible to find a gauge transformation $\left\{\mu_{i}= \pm 1 \mid i \in \mathbb{Z}^{d}\right\}$ such that the effective Hamiltonian is of the form

$$
\begin{aligned}
\mathscr{H}_{\text {eff }} & =-\sum_{\langle i, j\rangle} J_{i j}^{\text {eff }} S_{i} S_{i}+\text { higher body terms } \\
S_{k} & =\frac{1}{\left|\mathscr{C}_{k}\right|} \sum_{i \in \mathscr{C}_{k}} \mu_{i} \sigma_{i}
\end{aligned}
$$

with the $J_{i j}^{\text {eff }}$ predominantly ferromagnetic. By definition, this means that the original system is minimally frustrated. Note that this would imply that the block variables are ferromagnetically ordered, although the original variables are only ordered in the sense of $q_{\mathrm{EA}}>0$.

It is not clear to the authors whether such effects actually occur for some range of parameters. An affirmative answer would not only be of interest in its own right; it would also provide another potential realization of the RFIM. As before, an external field of uniform strength $h$ applied to the original system transforms into a random field

$$
H_{k}=h \sum_{i \in \mathscr{C}_{k}} \mu_{i}
$$

in the block system. Again, however, the additional effect of local correlations between the cluster couplings makes it unclear whether the purely ferromagnetic RFIM is a "good approximation."

Acknowledgements. We thank M. Aizenman, J. Bellissard, D. Huckaby, and S. Janowsky for helpful conversations related to the questions studied in this paper. We also thank the organizers of the Les Houches summer school for inviting us to participate in the school 
which was beneficial to our collaboration. J.T.C. and L.C. thank ETH (Hönggerberg and Zürich), and J.F. thanks the Institute for Advanced Study for hospitality. Finally, J.T.C. and L.C. thank the National Science Foundation for its support and the opportunities this has provided.

\section{References}

1. Griffiths, R.B., Lebowitz, J.L.: Random spin systems: some rigorous results. J. Math. Phys. 9, 1284 (1968)

2. van Enter, A.C.D., Griffiths, R.B.: The order parameter in a spin glass. Commun. Math. Phys. 90, 319 (1983)

3. Edwards, S.F., Anderson, P.W.: J. Phys. F 5, 965 (1975)

4. Peierls, R.: Proc Camb. Phil. Soc. 32, 447 (1936)

5. Griffiths, R.B.: Peierls proof of spontaneous magnetization in a two-dimensional Ising ferromagnet. Phys. Rev. 136, A 437 (1964)

6. Suzuki, M., Ikeda, H.: Magnetic specific heat of $\mathrm{Rb}_{2} \mathrm{CO}_{c} \mathrm{Mg}_{1-{ }_{c} \mathrm{~F}_{4}}$ : effect of dilution on a twodimensional Ising-like antiferromagnet. J. Phys. C11, 3679 (1978)

7. Cowley, R.A., Birgeneau, R.J., Shirane, G.: In: Order in strongly fluctuating condensed matter systems. Riste, T. (ed.). New York: Plenum 1980

8. Cowley, R.A., Birgeneau, R.J., Shirane, G., Guggenheim, H.I., Ikeda, H.: Spin fluctuations in random magnetic-nonmagnetic two-dimensional antiferromagnets. III. An Ising system. Phys. Rev. B 21, 4038 (1980)

9. Lubensky, T.C.: Ann. Israel Phys. Soc. Statphys. B 2, 216 (1978)

10. Lubensky, T.C.: In: Ill-condensed matter. Balian, R., Maynard, R., Toulouse, G. (eds.). Amsterdam: North-Holland 1979

11. Korenblit, I.Ya., Shender, E.F.: Ferromagnetism of disordered systems. Sov. Phys. Uspekhi 21, 832 (1978)

12. Stinchecombe, R.B.: In: Phase transitions and critical phenomena, Vol. 7. Domb, C., Lebowitz, J.L. (eds.). London: Academic Press 1983

13. Georgii, H.: Spontaneous magnetization of randomly dilute ferromagnets. J. Stat. Phys. 25, 369 (1981)

14. Aizenman, M., Chayes, J.T., Chayes, L., Fröhlich, J., Russo, L.: On a sharp transition from area law to perimeter law in a system of random surfaces. Commun. Math. Phys. 92, 19 (1983)

15. Lee, T.D., Yang, C.N.: Statistical theory of equations of state and phase transitions. II. Lattice gas and Ising model. Phys. Rev. 87, 410 (1952)

16. Falk, H., Gehring, G.A.: Correlation function and transition temperature bounds for bonddisordered Ising systems. J. Phys. C 8, L 298 (1975)

17. Olivieri, E.: Unpublished

18. Harris, A.B.: Upper bounds for the transition temperatures of generalized Ising models. J. Phys. C 7, 3082 (1974)

19. Bergstresser, T.K.: Rigorous upper and lower bounds on the critical temperature in Ising models with random, quenched, broken-bond disorder. J. Phys. C 10, 3831 (1977)

20. Griffiths, R.B.: Nonanalytic behavior above the critical point in a random Ising ferromagnet. Phys. Rev. Lett. 23, 17 (1969)

21. Fröhlich, J., Imbrie, J.Z.: Improved perturbation expansion for disordered systems: beating Griffiths singularities. Commun. Math. Phys. 96, 145 (1984)

22. Lebowitz, J.L.: GHS and other inequalities. Commun. Math. Phys. 35, 87 (1974)

23. Georgii, H.: On the ferromagnetic and percolative region of random spin systems. Preprint (1984)

24. Kesten, H.: The critical probability of bond percolation on the square lattice equals $1 / 2$. Commun. Math. Phys. 74, 41 (1980) 
25. Avron, J.E., Roepstorff, G., Schulman, L.S.: Ground state degeneracy and ferromagnetism in a spin glass. J. Stat. Phys. 26, 25 (1981)

26. Roepstorff, G.: The Peierls-Griffiths argument for disordered Ising systems. J. Math. Phys. 22, 3002 (1981)

27. Fishman, S., Aharony, A.: Random field effects in disordered anisotropic antiferromagnets. J. Phys. C 12, L729 (1979)

28. Chayes, L.: Thesis (Princeton 1983)

29. Russo, L.: Z. Wahrscheinlichkeitstheor. Verw. Geb. 43, 39 (1978)

30. Seymour, P.D., Welsh, D.J.A.: Ann. Discrete Math. 3, 227 (1978)

31. Kesten, H.: Percolation theory for mathematicians. Boston: Birkhäuser 1982

32. Hammersley, J.M.: Ann. Math. Statist. 28, 790 (1957)

33. Kesten, H.: Analyticity properties and power law estimates of functions in percolation theory. J. Stat. Phys. 25, 717 (1981)

34. Aizenman, M., Chayes, J.T., Chayes, L., Fröhlich, J., Russo, L.: Unpublished

35. Aizenman, M.: Absence of an intermediate phase for a general class of one component ferromagnetic models, preprint (1984) [see also, Aizenman, M. In: Statistical physics and dynamical systems, Proceedings Köszeg, 1984. Szasz, D., Petz, D. (eds.). Boston: Birkhäuser (in press)]

36. Griffiths, R.B.: Correlations in Ising ferromagnets. II. External magnetic fields. J. Math. Phys. 8, 484 (1967)

Kelley, D.G., Sherman, S.: General Griffiths' inequalities on correlations in Ising ferromagnets. J. Math. Phys. 9, 466 (1968)

37. Fortuin, C., Kasteleyn, P., Ginibre, J.: Correlation inequalities on some partially ordered sets. Commun. Math. Phys. 22, 89 (1971)

38. Harris, T.E.: Proc. Camb. Phil. Soc. 56, 13 (1960)

39. Kesten, H.: Surfaces with minimal weights and maximal flows: A higher-dimensional generalization of first passage percolation. Preprint (1985)

40. Kesten, H.: First-passage percolation and a higher-dimensional generalization. Preprint (1985)

41. Aizenman, M., Deylon, F., Soulliard, B.: Lower bounds on the cluster size distribution. J. Stat. Phys. 23, 267 (1980)

42. Kunz, H., Souillard, B.: Essential singularity in percolation problems and asymptotic behavior of cluster size distribution. J. Stat. Phys. 19, 77 (1978)

43. Russo, L.: Z. Wahrscheinlichkeitstheor. Verw. Geb. 56, 229 (1981)

44. Russo, L.: Z. Wahrscheinlichkeitstheor. Verw. Geb. 61, 129 (1982)

45. Imry, Y., Ma, S.-K.: Random-field instability of the ordered state of continuous symmetry. Phys. Rev. Lett. 35, 1399 (1975)

46. Aharony, A., Imry, Y.: Solid State Commun. 20, 899 (1976)

47. Aharony, A.: Tricritical points in systems with random fields. Phys. Rev. B 18, 3318 (1978)

48. Aharony, A.: Spin-flop multicritical points in systems with random fields and in spin glasses. Phys. Rev. B 18, 3328 (1978)

Communicated by A. Jaffe

Received January 7, 1985; in revised form April 5, 1985 
